\title{
Effect of Simulated Scalloped Ice on the Aerodynamics of a Swept-Wing at Low-Reynolds Number
}

\author{
Navdeep Sandhu*, Mohamad Reza Soltani ${ }^{\dagger}$, Michael B. Bragg*, Christopher W. Lum ${ }^{\S}$, \\ University of Washington, Seattle, WA, 98195, USA \\ and Brian S. Woodard ${ }^{\mathbb{I}}$ \\ University of Illinois at Urbana-Champaign, Urbana, Illinois, 61801, USA \\ and Andy P. Broeren ${ }^{\#}$ \\ NASA John H. Glenn Research Center, Cleveland, Ohio, 44135 USA \\ and Sam Lee** \\ Vantage Partners, LLC, Cleveland, OH, 44135 USA.
}

This paper studied the aerodynamic effects of a single scalloped ice accretion and two lower fidelity ice-shape simulations. These data were compared to the aerodynamics of a clean $8.9 \%$ scale CRM65 semispan wing model at a Reynolds number of $1.6 \times 10^{6}$. The clean wing experienced an aggressive, tip-first stall and showed a small, strong leading-edge vortex at lower angle-of-attack while the iced cases showed larger, seemingly weaker leading-edge vortices at similar angles. The size of these vortices is larger for the low-fidelity ice shape. The stall pattern for the iced cases was also tip-first, but more gradual than the clean wing. The high-fidelity ice shape produced streamwise flow features over the upper surface of the wing due to flow moving through gaps that exist in the ice shape geometry that disrupted the formation of the leading-edge vortices, changing the aerodynamics of the wing. These gaps do not exist in the low-fidelity shape. The low-fidelity scallop ice shape was non-conservative in its aerodynamic penalties compared to the full high-fidelity case.

\section{Nomenclature}

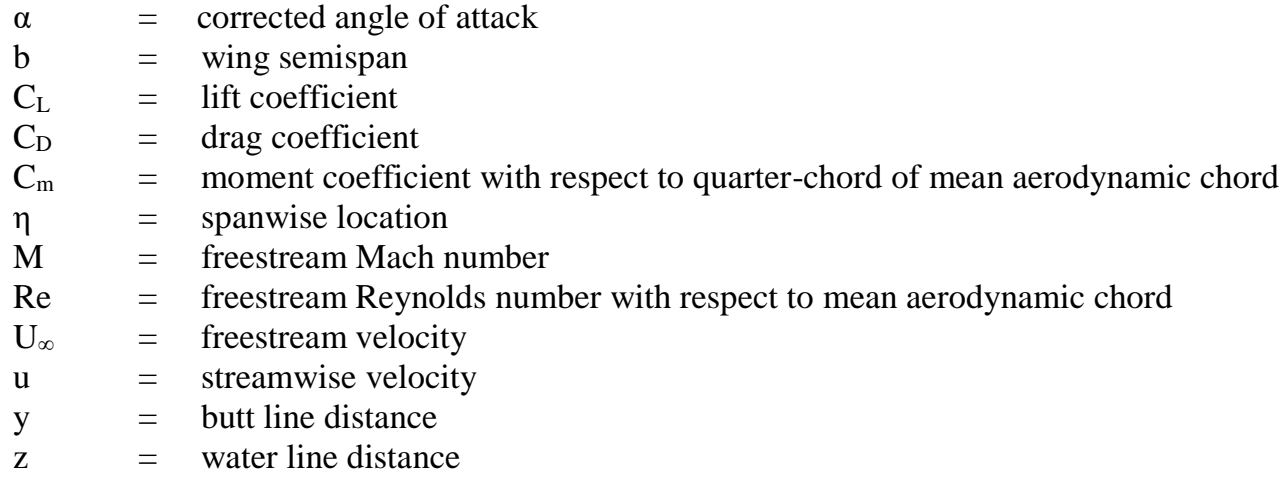

\footnotetext{
* Research Assistant, William E. Boeing Department of Aeronautics and Astronautics; Guggenheim Hall Room 211, Box 352400, Seattle, WA 98195-2400, AIAA student member.

${ }^{\dagger}$ Visiting Professor, William E. Boeing Department of Aeronautics and Astronautics; Guggenheim Hall Room 211, Box 352400, Seattle, WA 98195-2400.

${ }^{\ddagger}$ Frank and Julie Jungers Dean, College of Engineering, and Professor, William E. Boeing Department of Aeronautics and Astronautics; Loew Hall Room 371, Box 352180, Seattle, WA 98195-2180, AIAA fellow.

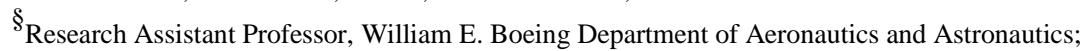
Guggenheim Hall Room 211, Box 352400, Seattle, WA 98195-2400, AIAA member.

${ }^{\mathbb{I}}$ Research Associate, Department of Aerospace Engineering, 306 Talbot Lab, 104 S. Wright St., AIAA member.

\# Research Aerospace Engineer, Icing Branch, 21000 Brookpark Rd., Associate Fellow AIAA.

*** Research Engineer, Icing Branch, 21000 Brookpark Rd., Senior Member AIAA.
} 


\section{Introduction}

The effects of full-span ice shapes on the aerodynamic performance of swept wings is not currently well understood and can lead to adverse aerodynamic performance that is not well described in the literature. Previous published research has mainly focused on 2D aerodynamic effects of ice accretion on airfoils and straight wings. Bragg et. al [1] reviewed the extensive 2D aerodynamic effects of ice accretion on airfoils and classified ice accretions by their aerodynamic effect. It is important to extend our understanding of iced-wing aerodynamics to include swept wings to improve the design, testing, and certification of aircraft. Toward this objective, NASA, FAA and ONERA with their academic partners and Boeing have engaged in a research program with one of its goals to improve the fidelity of experimental and computational methods for swept-wing ice formation and the resulting aerodynamic effect [2].

The Common Research Model (CRM) is a publicly available swept-wing geometry that is representative of current, modern-design civilian transport airplanes. After reviewing the available options, a 65\% scale Common Research Model (CRM65) geometry was selected [2,3] for this research program. To generate the full-scale artificial ice shapes for aerodynamic testing, ice accretions were generated in the Icing Research Tunnel (IRT) at NASA Glenn Research Center utilizing hybrid models representing the $\eta=20 \%, \eta=64 \%$, and $\eta=83 \%$ spanwise locations (referred from here on as the inboard, midspan, and outboard stations) [4]. The models had full-scale leading edges with a redesigned aft section and reduced chord length to fit within the IRT test section with acceptable blockage. The hybrid models matched the full-scale wing leading-edge geometry allowing the accretion of full-scale ice accretions in the IRT. This was accomplished experimentally by matching the attachment line locally via model flap deflection. The procedure used to design these hybrid models is described in more detail by Fujiwara $[5,6,7,8]$.

Using the full-scale ice accretions digitized from the IRT tests via laser scanning, ice accretion simulations were constructed for full-span swept-wing aerodynamic testing [9]. Low-Reynolds number testing was conducted in the Wichita State University's Walter H. Beech tunnel and high-Reynolds number testing at the ONERA F1 tunnel. Initial results from the low-Reynolds number test have been presented by Broeren et. al. [10], Camello et. al. [11], and Lum et. al [12]. Camello and coauthors provided a preliminary overview of the iced-swept-wing results to date from the low-Reynolds number testing. Using balance data, pressure coefficient data, flow visualization and wake data they focused on two ice accretions and their different fidelity simulations tested. The low fidelity results were not conservative in producing aerodynamic penalties and preliminary flowfield analysis suggested differences in the flowfields, especially during the stalling process. To better understand the effect of fidelity on ice accretion aerodynamic simulation, and thus to improve our ability to accurately produce swept-wing performance with ice, these flowfield and aerodynamic differences need to be better understood.

The aerodynamic behavior of a swept-wing is complicated due to the complex flowfield over the wing and its variations with angle of attack. Sweep affects the aerodynamic characteristics of a wing by altering the spanwise distribution of boundary-layer growth over the wing surface, flow separation, and the onset of stall. These effects and more were explored by Lynch and Khodadoust [13]. Depending on the wing-sweep angle, the spanwise component of velocity may create a spiral vortex, similar to that of a delta wing at moderate $\alpha$. Poll [14] determined that these vortices can form over the entire span of swept-wings with moderate sweep angles due to the rolling up of shear layer that leaves the airfoil surface at the primary separation line very near the leading edge of the wing. Poll also determined that part-span vortices can form if the shear layer re-attaches in the inboard of the wing (creating a short bubble instead) but does not re-attach on the outboard regions due to higher loading in this region. The portion of the shear layer that does not re-attach to the wing rolls up to create the vortex. This vortex will alter the pressure distribution considerably and may increase the local stall angle of attack. There is also a drag increase associated with higher lift.

Flowfield complexity increases drastically with the addition of an ice shape on the leading-edge of the wing. It has been shown repeatedly that leading-edge ice accretions will degrade aerodynamic performance $[15,16]$. Both Broeren et al. [15] and Diebold et al. [16] have shown that the effect of $M$ and Re is reduced when considering a swept-wing with leading-edge ice. They have also shown that these ice shapes will alter the formation of leading-edge separation over the wing. In turn, this changes stall progression from a leading-edge type to a trailing-edge type similar to that of a thin airfoil. The model used in this study includes twist, not considered by Broeren et al, and at higher $R e$ than the exploration by Diebold et al.

This paper again considers the low-Reynolds number data on the swept wing with and without ice expanding on the analysis on Camello et al. [11]. By further analyzing the flowfield about a single scalloped ice accretion, and its various fidelity ice-shape simulations, the goal is to better understand how the ice shape affects the swept-wing aerodynamics and how the fidelity of the simulated shape impacts the accuracy of the resulting aerodynamic simulation and integrated performance. 


\section{Experimental Methods}

The low-Reynolds number aerodynamic testing of the full-span artificial ice shapes was performed in the $7 \times 10$ ft. Beech Memorial Wind Tunnel at Wichita State University, an atmospheric, closed-return tunnel. An 8.9\% scale CRM65 semispan wing model was used for this testing and a summary of the subscale model parameters and dimensions are shown in Table 1 and Fig. 1.

The model was designed to test artificial ice shapes and includes a removable leading-edge. The main wing and clean leading edge were machined from aluminum. The iced, reusable leading edges were rapid prototyped using stereolithography. The removable leading-edge segments were attached to the main wing model for aerodynamic testing. The clean, aluminum leading-edge model was tested with a variety of boundary-layer trip configurations on both the upper and lower surfaces of the wing to provide baseline comparison data. This model was tested at the conditions summarized in Table 2 with clean and a variety of leading-edge ice-shape configurations. Additional diagrams and images of the model can be found in Broeren [10].

The model was constructed with several rows of pressure taps to acquire surface pressure data. Figure 2 shows a picture of the wing with the pressure tap rows marked. Force-balance data were acquired as well over a range of angles of attack and corrected for wall interference. These data and their respective uncertainty are described in detail in Broeren et al $[10,17]$. A summary of the uncertainty of these data are given in the appendix.

Multiple ice accretions were generated in the IRT and were used to develop full-span artificial ice shapes tested in the tunnel at Wichita State University. Details about this process are presented by Camello et al [9]. The aerodynamics of the maximum scallop ice shape are studied in detail in this paper. An important part of the evaluation of the aerodynamics of the ice shapes on the swept wing model is the effect of ice-shape fidelity. Three fidelities of the maximum scallop ice shape were created and have been tested to date: high-fidelity, low-fidelity 3D smooth, and lowfidelity 3D smooth with 60-grit roughness. The high-fidelity model is one that is the result of a laser scan of three spanwise stations of ice accretions (inboard, midspan, and outboard) that have then been interpolated between to generate a full-span leading-edge ice shape. The low-fidelity 3D smooth ice shape geometries were created by lofting through a series of $2 \mathrm{D}$ cuts along the span of the high-fidelity ice shape geometry.

Two different flow visualization methods were used during the tests. Fluorescent mini-tuft data were collected during aerodynamic performance sweeps for many of the model configurations. 0.0019-inch diameter fluorescent monofilament tufts were attached to the model surface with cyanoacrylate adhesive in streamwise rows, the tufts were illuminated by a remote flash with a UV black light filter so they could be photographed at select angles of attack. The second visualization method used was surface-oil flow visualization. A mixture of mineral oil and fluorescent dye was applied to black contact paper on the wing surface with sponge paint rollers. The tunnel was then ramped up to the desired speed for a total run time of two minutes from fan on to fan off. Again, the test section was illuminated by a remote flash with a UV black light filter so the model could be photographed with the surface oil. These visualization methods can be used to detect three-dimensional flow characteristics present such as separated flow and vortices. More detail on the wind tunnel model and experimental methods can be found in Broeren et al [10].

Wake surveys using five-hole probes were acquired for a limited set of ice shape and angle of attack conditions. This technique was described by Lum et al [12]. The technique has been shown to provide reasonable quantitative measurements of lift and drag, including their spanwise distribution. The wake data are particularly useful in visualizing the large vortices shed into the wake during the swept-wing stalling process.

Note that in previous studies using these data $[10,11,12], \alpha$ was corrected in a different manner. In this paper, the correction used for $\alpha$ results in a decrease of approximately $0.5 \mathrm{deg}$. in $\alpha$ for the same cases when compared to previous studies using the data. More details on this change are given by Lee et al [18]. 
Table 1. Summary of $8.9 \%$ scale CRM65 semispan wing model parameters.

\begin{tabular}{|l|c|}
\hline \multicolumn{1}{|c|}{ Wing Parameter } & Value \\
\hline Span & $5.00 \mathrm{ft}$. \\
\hline Mean Aerodynamic Chord & $1.39 \mathrm{ft}$. \\
\hline Area & $6.01 \mathrm{ft}^{2}$ \\
\hline Aspect Ratio & 8.30 \\
\hline Taper Ratio & 0.23 \\
\hline Root Chord & $2.25 \mathrm{ft}$. \\
\hline Tip Chord & $0.52 \mathrm{ft}$. \\
\hline Washout & $8.2 \mathrm{deg}$. \\
\hline 1/4-chord sweep angle & $35 \mathrm{deg}$. \\
\hline Leading-edge sweep angle & $37.2 \mathrm{deg}$. \\
\hline
\end{tabular}

Table 2. $M$ and $R e$ conditions tested.

\begin{tabular}{|c|c|}
\hline $\boldsymbol{M}$ & $\boldsymbol{R e}$ \\
\hline 0.09 & $0.8 \times 10^{6}$ \\
\hline 0.17 & $1.6 \times 10^{6}$ \\
\hline 0.26 & $2.4 \times 10^{6}$ \\
\hline
\end{tabular}

\section{Results and Discussion}

Force balance, surface pressure, and mini-tuft flow visualization data were collected for each of the three Reynolds numbers and Mach number combinations on all the model and ice shape configurations. A summary of the lift, drag, and moment results for the clean wing for the three Reynolds number/Mach number combinations tested is shown in Fig. 3. Results for the iced cases are quantified by Broeren et al [17]. The results on the clean model show very similar results at the two higher $R e$ and $M$ combinations while the lower $R e$ and $M$ data deviate more significantly at the higher angles of attack. The iced data show even less $R e$ and $M$ dependence in this range. An analysis of the effect of $R e$ and $M$ will be presented in detail in another paper at the conference by Broeren et al [19]. Lee et al [18] are also examining these effects and show that the effect of both $R e$ and $M$ for the high-fidelity maximum scallop shape is smaller than that for the clean wing, which shows a greater difference in aerodynamics with a change in $R e$ than a change in $M$. This paper will focus on the results for a single combination of $R e$ and $M, R e=1.6 \times 10^{6}$ and $M=0.17$, as the primary goal of this paper is to better understand the swept-wing aerodynamics with ice accretions and how this is affected by ice shape fidelity. A comparison of the aerodynamic data for the high-fidelity maximum scallop ice shape to the two low-fidelity maximum scallop ice shapes as well as the clean wing is summarized in Fig. 4. In this plot there is significant aerodynamics performance differences between the high-fidelity ice shape and the two 3D smooth representations, with and without grit roughness. The effect of adding the grit roughness on the 3D smooth low-fidelity roughness is small. This was also considered by Camello et al. [11] (Camello refers to this same 3D smooth lowfidelity model as the 2D smooth model). Thus, this paper will focus on the clean wing, the 3D smooth maximum scallop ice shape, and the high-fidelity maximum scallop ice shape.

Computer-generated geometry for the 3D smooth maximum scallop ice shape and the high-fidelity maximum scallop ice shape are shown at select spanwise points in Fig. 5. The geometry for the 3D smooth maximum scallop ice shape is based on the high-fidelity maximum scallop shape. Not all high-fidelity ice shape features are recreated in the smooth ice shape. The process of the creation of the ice shape is detailed by Camello et al [9]. The geometry is viewed from the lower surface, with the root to the left of the image and the wing tip to the right. The geometry for both ice shapes changes with span. For the 3D smooth ice shape, there is a large variation near the midspan. The shape changes curvature rapidly in this region. The high-fidelity shape has scallops that extend out from the leading edge. The scallops are the three-dimensional ice features that repeat in the spanwise direction and result in gaps for airflow 
to move between the scallops in primarily a streamwise direction. The width of these gaps, along with the width of the scallops, change with span as well. For inboard and outboard sections of the wing, the ratio of scallop width to gap width is close to 1 . This ratio increases near the midspan. Scallop width and gap width are plotted versus span in Fig. 6. A physical view of this change is shown in Fig. 5. This variation in the scallops and the resulting gaps will be shown in a later section to impact the flowfield.

\section{A. Clean Wing}

The aerodynamics of swept wings are made complex due to a combination of several 3D effects. Some of these effects become apparent when observing the stall progression of the wing with increasing angle of attack. The progression of 3D effects and flow separation with increasing $\alpha$ is seen most clearly using flow visualization. Figure 7 shows mini-tuft imagery for the clean wing for $\alpha=4.31$ deg., $\alpha=8.50$ deg., $\alpha=10.57$ deg., and $\alpha=12.63$ deg. In Fig. 8, surface oil flow is shown also for $\alpha=4.31$ deg., $\alpha=8.50$ deg., $\alpha=10.57$ deg., and $\alpha=12.63 \mathrm{deg}$.

The clean swept wing flowfield at increasing angle of attack is characterized by the formation of a leading-edge separation vortex, strong spanwise flow that develops on the surface near the trailing edge, and eventually large-scale flow separation. These features are well known and are described in the classic paper by Poll [14] and have been identified for this geometry in several earlier papers $[10,11,16]$. Here we see in the mini-tuft images and oil flow these same features.

At $\alpha=4.31 \mathrm{deg}$. the mini-tufts are in the streamwise direction root to tip There is no motion-blur of the tufts to indicate any unsteady or separated flow. As $\alpha$ increases to 8.50 deg., there is some outboard movement of the tufts near the trailing edge of the wing. The tufts indicate a spanwise flow direction, from the wing root to the wing tip. The oil flow visualization at low $\alpha$ shows a leading-edge separation vortex. The vortex is small and not visible in the minitufts. Primary separation occurs on the leading edge, the flow reattaches a short distance downstream, and the reverse flow experiences a second, secondary separation, right behind the initial separation point. These features are identified in the oil as we move downstream on the leading edge, where in this upper surface view separation occurs but can't be easily seen, followed by a bright spanwise pooling of oil which is the secondary separation, and then a black region relatively void of oil, which is just ahead of reattachment where the oil is scrubbed forward to form the bright secondary separation line. This type of flowfield has been extensively studied in 2D and described by many authors including for the icing case by Bragg et a. [1]. The primary difference here, due to the spanwise gradients, is the rolling up of this separation into a spanwise vortical, or vortex flow. Kerho et al. [20] show this feature clearly on a swept iced wing using LDV flowfield measurements and helium bubble flowfield tracers.

For $\alpha=10.57$ deg., the mini-tufts in Fig. 7 show spanwise flow near the trailing edge from root to tip. On the outboard section of the wing, there are reversed mini-tufts near the wing tip identifying separation occurring near the wing tip. This was also seen by Broeren [10]. While this wing has significant washout (over $8 \mathrm{deg}$.) intended to reduce the tendency of swept wings to experience tip stall, the tip Reynolds number is very low for these tests and well below the design value. The classic explanation involves the boundary layer over the outboard sections of the wing to thicken due to spanwise flow, coupled with the adverse pressure to cause early tip separation. The mini-tuft imagery for $\alpha=$ $12.63 \mathrm{deg}$. shows that separation continues to occur from the outboard to the root of the wing. Though the change in $\alpha$ is small (approximately $2 \mathrm{deg}$.), the separation progresses quickly, and continues to do so until $\mathrm{C}_{\mathrm{L}, \max }$ occurs at approximately $\alpha=13.5 \mathrm{deg}$.

The oil flow imagery shows the tip separation at $\alpha=10.57 \mathrm{deg}$. and it grows in extent as $\alpha=12.63 \mathrm{deg}$. Near the wing tip, the leading-edge vortex in this $\alpha$ range expands rapidly. This expansion likely contributes to the separation occurring in this region, along with the spanwise flow. This separation region grows large as $\alpha$ increases to $12.63 \mathrm{deg}$.

Figure 9 shows the upper-surface $C_{p}$ distributions for the clean wing at $\alpha=4.31$ deg., $\alpha=8.50$ deg., $\alpha=10.57$ deg., and $\alpha=12.63 \mathrm{deg}$. for the five streamwise rows on the wing. Here the root $\mathrm{Cp}$ distributions are on the right side of each the plot and the tip on the left. For low $\alpha$, the pressure coefficient peaks (low pressure) near the leading edge, indicates a large amount of suction on the upper surface, leading edge of the wing. The pressure coefficient distributions show suction on the upper surface continuing to increase as $\alpha$ increases to $8.50 \mathrm{deg}$. The mini-tuft and oil flow imagery show separation near the wing tip for $\alpha=10.57 \mathrm{deg}$. However, there are no pressure taps in this region (for $y / b>0.9$ ), and thus this separation is not seen clearly in the $C_{p}$ distribution. The tip-most pressure row shows a slight pressure increase near the leading-edge, which may be an effect of the separation in this region. The separation is seen more clearly for $\alpha=12.63 \mathrm{deg}$. The outer two pressure rows show $\mathrm{C}_{\mathrm{p}}$ has increased and is relatively 
flat along the local chord for these regions indicating flow separation. These two rows correspond to $\mathrm{y} / \mathrm{b}=0.81$ and $\mathrm{y} / \mathrm{b}=0.9$, corresponding to the region where separation has occurred for this $\alpha . \mathrm{C}_{\mathrm{L}, \max }$ for the clean wing occurs at approximately $\alpha=13.5 \mathrm{deg}$. At this point, the lift coefficient drops sharply, the drag coefficient increases rapidly, and the change in $\mathrm{C}_{\mathrm{m}}$ with $\alpha$ becomes positive. The nose-up, positive, change in pitching moment with $\alpha$ is a classic effect of swept wings experiencing tip stall and approaching $\mathrm{C}_{\mathrm{L}, \max }$. At this point, flow over most of the wing has separated. The only non-separated region is near the root again contributing to the nose-up pitching moment.

All data seems to agree with respect to the phenomena occurring on the clean wing as $\alpha$ increases. Spanwise flow near the trailing edge, leading-edge separation and the formation of a leading-edge vortex, and then tip stall which moves inboard leading to maximum lift, large drag rise, and nose-up pitching moment. The clean wing shows indications of an aggressive tip-first stall that occurs beginning at $\alpha=10.57 \mathrm{deg}$., with $\mathrm{C}_{\mathrm{L} \text {,max }}$ occurring at $\alpha=13.5$ deg. Prior to this point, a leading-edge vortex forms along the span of the wing that later diffuses rapidly, contributing to separation of the flow. Flow separation is also caused by the spanwise flow moving from the root of the wing to the tip thickening the boundary layer and creating a strong adverse pressure gradient.

\section{B. 3D Smooth Maximum Scallop}

Two low-fidelity versions of the maximum scallop shape were tested as part of this wind tunnel program. They are both geometrically based on the high-fidelity maximum scallop ice shape. One of the shapes had roughness manually applied to simulate roughness effects associated with the high-fidelity ice shape. Figure 4 shows the change in $C_{L}$ and $C_{D}$ with $\alpha$ for both shapes. Aside from a small shift in magnitude, the smooth and rough shapes have nearly identical aerodynamic performance. Thus, for this $R e$ and $M$, the effect of roughness is considered negligible, and only the 3D smooth maximum scallop shape without grit will be considered for comparison to the high-fidelity maximum scallop shape and the clean wing.

Examining Fig 4, the aerodynamic performance of the iced versus clean wing shows reduced lift-curve slope and reduced maximum lift. Pitching moment is more nose up and breaks at a much lower angle of attack than the clean wing. At angles of attack less than 6 degrees, the drag is significantly higher than the clean for the 3D smooth shapes, and even higher for the high-fidelity shapes. First let's examine the flowfield of the swept wing with the 3D smooth ice shape and compare to that of the clean wing.

The differences in the aerodynamics between the 3D smooth shape and the clean case can be examined using the flow visualization. Mini-tuft imagery for the 3D smooth maximum scallop ice shape at $\alpha=4.30$ deg., $\alpha=6.40$ deg., $\alpha=8.46$ deg., and $\alpha=10.50 \mathrm{deg}$. is shown in Fig. 10. (Note that some of these angles of attack are different than the clean case in Fig. 7. where $\alpha$ 's of 4.31, 8.50, 10.57, and 12.63 degs. are shown.) At $\alpha=4.30$ deg. evidence is seen in the tufts of leading-edge separation and leading-edge vortex formation in the midspan region. At this angle of attack the clean wing (Fig. 7) saw no separation or vortex present. There is also some motion blur of the tufts near the leading edge of the wing at approximately $y / b=0.5$, which is evidence of unsteady flow over the surface of the wing. As $\alpha$ increases to $6.40 \mathrm{deg}$. the tufts in Fig. 10 show that the separation region has grown rapidly over the outboard section of the wing. The leading-edge vortex starts farther inboard, and the spanwise flow near the trailing edge is extensive. Upon closer expansion of the tufts, two regions of leading-edge separation are seen divided by a row of mini-tufts near the midspan of the wing that is aligned with the freestream and a small region here of no, or reduced separation. This midspan region of reduced separation is likely due to a change in the 3D smooth ice shape geometry near this spanwise region. The curvature of the ice shape changes, as seen in Fig. 5 for the 3D smooth shape in the top image with the dotted line indicating the wing midspan.

The oil flow visualization for $\alpha=4.30 \mathrm{deg}$. and $\alpha=6.40 \mathrm{deg}$. is given in Fig. 11 . The oil flow imagery for $\alpha=4.30$ deg. shows a distinct reattachment line that results due to the separation and formation of the leading-edge vortex and corresponds well to the tuft data. The separation region for this $\alpha$ is much larger than that of the clean wing at a comparable $\alpha$. For $\alpha=6.40$ deg., the oil flow visualization confirms the significant separation seen in the tuft data. There is a region of oil accumulation near $\mathrm{y} / \mathrm{b}=0.5$ that is followed by a dark, oil-scrubbed region slightly outboard of this point. This likely correlates with a region of streamlined tufts seen for the same $\alpha$. This region likely lies between areas of separation. The existence of large recirculation regions in the 3D smooth case are likely the reason why the lift coefficient curve has a change in slope in the $\alpha$ range of 6 to 8 degrees and the drag coefficient also turns much larger in this $\alpha$ region 
Again, from the tuft data in Fig. 10, for $\alpha=8.46$ deg. the separation regions grow even larger, encompassing more of both the chord and span of the wing. The flow appears to be separated from the Yehudi break to the tip. The midspan region is no longer distinct. This trend continues for $\alpha=10.50$ deg., as flow separation moves toward the root of the wing. Note that no oil flow visualization data was collected for $\alpha=8.46$ and $\alpha=10.50 \mathrm{deg}$.

This flow separation pattern is seen in the $\mathrm{C}_{\mathrm{p}}$ distribution data shown in Fig. 12 for $\alpha=4.30$ deg., $\alpha=6.40$ deg., $\alpha$ $=8.46$ deg., and $\alpha=10.50$ deg.) Note that there are no pressure taps in the ice shape itself, and thus the pressure coefficient data point closest to the leading edge is further downstream than in the clean case. For the clean case, the first pressure tap is located at a local $\mathrm{x} / \mathrm{c}$ station of 0 for each pressure row. For both ice cases, the streamwise pressure row nearest the root begins at local $\mathrm{x} / \mathrm{c}=0.0075$. The streamwise pressure row nearest the tip begins at local $\mathrm{x} / \mathrm{c}=$ 0.05 . All other streamwise pressure rows begin at local $\mathrm{x} / \mathrm{c}=0.015$. The pressure coefficient distribution for the $3 \mathrm{D}$ smooth maximum scallop ice shape at $\alpha=4.30 \mathrm{deg}$. is similar to that of the clean wing at comparable $\alpha$. The suction peaks are slightly lower, but this is probably explained by the tap placement explained above. The separation near the leading edge is not clearly captured due to a lack of pressure taps in this region. As $\alpha$ increases to 6.40 deg., the pressure coefficient slightly increases in magnitude. That is, the pressure coefficient decreases and there is lower pressure over the upper surface of the wing. The suction peak increases on the two inboard stations while the center row sees reduced suction from its neighbors probably due to the geometry change and flow region seen in the oil and tuft flow. As $\alpha$ increases to 8.46 deg., and then 10.50 deg., the pressure coefficient over the four outboard pressure rows shows the effect of flow separation (reduced suction and little variation in $\mathrm{Cp}$ with chordwise location). Note that some data presented for the clean case is at different $\alpha$ than the ice shape cases, with data for the clean case presented at $4.31,8.50,10.57$, and 12.63 degs. The effect is most prominent for $\alpha=10.50 \mathrm{deg}$. However, the pressure coefficients in the first row show continued increase in suction and local lift as $\alpha$ increases. The changes that occur with increasing $\alpha$ are more gradual, as compared to the clean-wing case. As the wing begins to unload at high $\alpha$ due to separation on the outboard wing, it is seen in Fig. 4 that the change in $\mathrm{C}_{\mathrm{m}}$ with $\alpha$ becomes positive around $\alpha=7$ deg.

The streamwise velocity deficit data were gathered using the wake survey technique on the swept wing with the 3D smooth ice shape at $\alpha=4.30$ and 6.40 deg. Note that the wake, seen in Fig. 13, has some jaggedness associated with the edge of the wake. This is due to the technique used to find the wake, which is explained in more detail by Lum et al [12]. The dark red seen near the edge of the wake indicates that the wake streamwise velocity is almost equal to the freestream velocity. As the color changes from red to blue, the streamwise velocity decreases based on the scale given in the figure. For $\alpha=4.30$ deg., there is a clear discontinuity in the wake near a spanwise station of $y / b$ $=0.6$, which coincides with the mini-tuft imagery and oil flow visualization. At $\alpha=6.40 \mathrm{deg}$., two large wake features on either side of this discontinuity at $y / b=0.6$ are observed, corresponding to the separation regions seen in the oil flow visualization and thus interpreted to be large vortices shed in to the wake. The figure shows flow direction in the cross-flow plane as vectors. These vectors show the rotation of the flow. This corresponds to a change in the curvature of the ice shape, which occurs near $y / b=0.5$. This change in curvature is seen in a computer-generated render of the geometry in Fig. 5. One advantage of the wake survey technique over other data collection techniques is the ability to reduce the data collected to spanwise variations in lift coefficient and drag coefficient. These sectional coefficients give insight into the local aerodynamics at a given point on the wing. The process of this reduction is covered in more detail by Lum et al [12]. The spanwise lift and drag coefficients for $\alpha=4.30 \mathrm{deg}$. and $\alpha=6.40 \mathrm{deg}$. for the 3D smooth maximum scallop ice shape are shown in Fig. 14. There is waviness in the spanwise distribution for both the lift and drag coefficients. These features coincide with the large vortices shed into the wake seen in the streamwise velocity deficit data, as well as with the separation regions seen in both the mini-tuft and oil flow visualization data. Local maxima in sectional lift coefficient correlate with local maxima in drag coefficient. Thus, the drag increase in these regions is caused by vortex-induced drag, as it is associated with an increase in sectional lift coefficient.

Figure 15 shows a composite image of the growth of the leading-edge vortices for the 3D smooth maximum scallop ice shape for several $\alpha$. At $\alpha=2.20$ deg., there is a small vortex near the leading-edge. The incoming flow separates around the ice shape and reattaches further downstream. On the upstream side of the reattachment line, particles move toward the leading-edge where they encounter a high pressure gradient region and form a weak vortex. On a swept wing, this vortex moves toward the wing tip due to spanwise flow over the wing. The particles downstream of the attachment line move slightly toward the wing tip due to the spanwise component of velocity, but then the streamwise component of free stream velocity overcomes the spanwise component and the particles flow almost parallel to the chord. As $\alpha$ increases to 4.30 deg., the attachment line is moved further downstream, increasing the radius of the 
leading vortex. In addition, the leading-edge vortex is formed closer to the wing root, but its termination is similar to that of the vortex formed at $\alpha=2.20 \mathrm{deg}$. The vortices formed over the wing surface at $\alpha=6.40 \mathrm{deg}$. are different than those at lower $\alpha$. There are four different vortices over the wing surface at this $\alpha$ and only one terminates near the wing tip. The other three terminate over the wing surface inboard of the wing tip due to the large pressure gradient over the wing surface caused by spanwise flow as well as wing twist, which causes variations in the vortex strength along the wing span. Note that at low $\alpha$, wing twist did not have a significant effect on the vortex strength along the wing span, but at higher $\alpha$ it may have some effect. At $\alpha=6.40$ deg., the flow over the wing surface is on the verge of separation, as seen by the sporadic vortices and their termination over the surface. For $\alpha=7.44$ deg., the attachment line has moved to mid-chord and vanishes prior to reaching the wing tip. The attachment line and the vortex shape both are similar to that of the clean wing. Therefore, it would be expected for flow to separate at $\alpha$ greater than $7.44 \mathrm{deg}$. This is the case, which is evident from Fig. 4 and is supported by the $C_{p}$ data as well. Thus, the formation of vortices for the 3D smooth maximum scallop shape is caused by the presence of the ice shape. The vortices themselves are different from those formed on the clean wing.

For $\alpha$ between 0 deg. and 2 deg., the lift coefficient for the 3D smooth maximum scallop ice shape matches well with the high-fidelity shape. This also true for the trend in drag coefficient for $\alpha$ less than 6 deg., though the magnitude of $\mathrm{C}_{\mathrm{D}}$ for the 3D smooth shape is much lower. The 3D smooth maximum scallop ice shape is associated with large leading-edge vortices, seen as recirculation regions in the oil flow visualization and mini-tuft data. Streamwise velocity deficit data show that the wake behind the wing with the 3D smooth ice shape attached is large and indicates the existence of the large recirculation regions over the top surface of the wing. Spanwise sectional lift and drag coefficients show regions of local maxima in lift and drag associated with vortices that were shed into the wake. Pressure coefficient distribution data as well as $C_{L}, C_{D}$, and $C_{m}$ data show that the change in the aerodynamics over the wing is somewhat gradual until high $\alpha$. Separation over the wing occurs sooner than for the clean wing but is not as rapidly progressing.

\section{High-Fidelity Maximum Scallop}

The high-fidelity maximum scallop ice shape has 3D features that significantly alter the aerodynamics of the wing. The aerodynamic performance is shown in Fig. 4. The high-fidelity maximum scallop shape lift performance is dissimilar to the 3D smooth shapes. The lift coefficient is lower for the high-fidelity shape than all other cases for $\alpha$ greater than $2 \mathrm{deg}$. The lift curve becomes nonlinear significantly earlier than the 3D smooth. In the $\alpha$ range from 0 to $6 \mathrm{deg}$. the pitching moment is more positive. The drag coefficient is much higher for the high-fidelity shape as compared to the 3D smooth for an $\alpha$ range of -6 to $6 \mathrm{deg}$.

Mini-tuft imagery for the high-fidelity maximum scallop ice shape case at $\alpha=4.27$ deg., $\alpha=6.35$ deg., $\alpha=8.42$ deg., and $\alpha=10.47 \mathrm{deg}$. is shown in Fig. 16. These are nominally the same angles of attack as Fig. 10 for the 3D smooth mini-tuft images. At $\alpha=4.27 \mathrm{deg}$. the high-fidelity flow is very similar to the 3D smooth at $\alpha=4.30 \mathrm{deg}$. Some spanwise flow develops near the trailing edge of the wing however the 3D smooth sees some leading -edge separation near the midspan that is not seen in the high-fidelity case. As $\alpha$ increases to 6.35 deg., the differences between the mini-tuft data for the high-fidelity maximum scallop ice shape and the 3D smooth maximum scallop ice shape are more apparent. On the high-fidelity maximum scallop ice shape tufts near the midspan leading-edge of the wing are indicative of the initiation of separation in this region. However, the 3D smooth maximum scallop ice shape has many more moving tufts, as the separation at a comparable $\alpha$ is much larger for that case.

The mini-tuft imagery at $\alpha=8.42 \mathrm{deg}$. show separation has occurred over a good portion of the outboard section of the wing. The separation region covers from a spanwise station of approximately $y / b=0.2$ to the wing tip. The mini-tuft imagery is similar for the high-fidelity ice shape case and the 3D smooth maximum scallop, which both seem to have separated flow from a spanwise station of approximately $\mathrm{y} / \mathrm{b}=0.2$ to the wing tip. As most of the flow over the wing separates, the ice shapes have less influence over the flow. This is likely why the high-fidelity ice shape and the 3D smooth ice shape converge in $C_{D}$ and $C_{m}$ for high $\alpha$.

Figure 17 shows oil flow visualization for $\alpha=4.27 \mathrm{deg}$. and $\alpha=6.35 \mathrm{deg}$. The oil flow visualization looks very different than the 3D smooth images in Fig. 11. Extensive streamwise features, which Camello et al. [11] identified as streamwise vortices, are seen. These features are not present on the 3D smooth shape. While the features may contain streamwise vorticity, a slightly different mechanism is proposed here. The high-fidelity maximum scallop shape is highly 3D and contains ice scallops that repeat spanwise and are separated or connected by regions of thinner ice accretion, shown in Fig. 5. The sizes of these scallops and the gaps between them are given in Fig. 6. Regions where the ratio between the size of the scallops and gaps are greater than 1 , such as between $y=22 \mathrm{in}$. and $\mathrm{y}=30 \mathrm{in}$., 
is where the streamwise features disappear in the oil flow visualization. For spanwise locations less than 22 in. and greater than $30 \mathrm{in}$., the ratio of scallop width to gap width is near or below 1. In these regions, the ice shape is not "solid" to the oncoming flow as is the 3D smooth shape that was formed from the maximum extent or thicknesses on the measured scallop shape. As described by Camello, Lee, et al. [9], the maximum scallop ice simulation tested was generated from three measured accretions of limited span and then morphing was used to generate a shape between these stations. Thus, the pattern of scallops and valleys repeats across the span changing slowly as it moves spanwise due to the morphing process. The dark streamwise regions correspond to these valleys between the scallop protuberances and allow relatively high-velocity air to flow through the ice shape and, due to the high shear, scrub off the oil exposing the black wing surface below. Thus, they act something like "jets" generated through the ice simulation. The bright streamwise regions are regions of reduced shear between the jets. There is some evidence of the oil streaks merging as they flow downstream that may well be the result of streamwise vortices, more research is needed to fully characterize this very complex 3D flow in this region. This flow feature is thought to be responsible for the differences in the swept-wing aerodynamics of the high-fidelity shape versus the simpler 3D smooth shape.

As $\alpha$ increases to 6.35 deg., oil flow visualization data indicates that the jet features are diminished near the midspan of the wing. This effect is likely due to the change in geometry of the ice shape itself. Near the midspan of the highfidelity maximum scallop ice shape, the gaps between the scallops decrease in size. A computer-generated rendering of this difference is shown in Fig. 5, image c). This decrease in gap size, coupled with increased $\alpha$, reduced the flow through the gaps in the ice in favor of moving around the leading-edge ice accretion instead. This is further evidenced by the existence of a flow separation region in the area where the jets disappear. The oil flow visualization shows a clear reattachment line from the separation region downstream of the ice shape. It is likely that the jets closer to the wing tip and near the root are preventing the flow from separating, as they would re-energize flow in this region by injecting higher energy flow. Further evidence of the flow separation and reattachment near the centerline is the apparent beading and concentration of oil just aft of the ice shape and ahead of the reattachment line in Fig. 17, $\alpha=$ $6.35 \mathrm{deg}$. This beading represents the secondary separation line inside the overall leading-edge separation vortex. Indicative of the complex flow in this region, the line appears to be interrupted periodically, probably by some small flow through the ice shape.

Figure 18 shows the $C_{p}$ distribution data for $\alpha=4.27$ deg., $\alpha=6.35$ deg., $\alpha=8.42$ deg., and $\alpha=10.47 \mathrm{deg}$. Note that there is no pressure data taken within the ice shape itself, similar to the 3D smooth shape case. For $\alpha=4.27$ deg., the magnitude of the peak pressure coefficient aft of the leading edge is lower than both the clean case and the 3D smooth shape case. This coincides with $C_{L}$ data that show a lower lift coefficient for the high-fidelity maximum scallop shape than either the 3D smooth shape or the clean wing. The magnitude of the pressure coefficient increases as $\alpha$ increases to $6.35 \mathrm{deg}$. As there are no pressure taps in the ice shape, the small leading-edge separation that exists at this $\alpha$ is not picked up by the $\mathrm{C}_{\mathrm{p}}$ distribution data. The pressure coefficient for $\alpha=8.42 \mathrm{deg}$. shows reduced leadingedge suction on the outboard sections foreshadowing the coming tip stall. At this $\alpha$ the 3D smooth is already showing significantly reduced lift and constant pressures over the tip section indicating tip stall as seen in the tufts. For $\alpha=$ 10.47 deg., the pressure coefficients between the two fidelities of the maximum scallop shape are nearly identical in the outboard and midspan regions. The pressure coefficients nearest the root still have a distinct peak in the 3D smooth case that does not exist for the high-fidelity shape case, which is likely why the 3D smooth shape case has a higher lift coefficient at this $\alpha$ and above. As the rest of the wing is already experiencing stall for both the 3D smooth shape and the high-fidelity shape, the difference in aerodynamic performance is likely a function of the phenomena at the wing root. The increased suction peak at the root for the 3D smooth shape compared to the high-fidelity shape also explains the why the change in $\mathrm{C}_{\mathrm{m}}$ with $\alpha$ is more positive for the $3 \mathrm{D}$ smooth case than it is for the high-fidelity case. This suction peak is ahead of the moment reference at the quarter-chord point of the mean aerodynamic chord and thus causes a nose-up moment.

The streamwise velocity deficit in the wake of the wing for the high-fidelity maximum scallop ice shape is shown in Fig. 19. For $\alpha=4.27 \mathrm{deg}$., the wake of the wing is consistent and thick, in contrast to the 3D smooth ice shape wake, Fig. 13, where the wake varied in thickness at different spanwise locations. The 3D smooth wake has a clear discontinuity centered at $\mathrm{y} / \mathrm{b}=0.55$ probably due to the ice shape discontinuity discussed earlier. There are vortices on either side of this discontinuity, evidenced by the cross-flow vectors that surround regions of low streamwise velocity. In general, the 3D smooth wake varies widely spanwise. The high-fidelity wake is very uniform spanwise and may be a result of the stabilizing tendencies of the jet/vortex flow produced by the scallops. The high-fidelity wake has more low-velocity flow in the wake as compared to the 3D smooth, reflective of the higher drag especially 
for the $\alpha=4.27$ deg. case. At the lower angles of attack the losses the flow experiences flowing through the scallop protuberances explain the lower wake velocity and higher drag. At higher angles of attack this is probably balanced by the overall flow separation which eventually dominates the drag. At $\alpha=4.47 \mathrm{deg}$., but especially at $\alpha=6.35 \mathrm{deg}$., closely placed and periodic regions of high velocity loss are seen in the center of the wake. They appear in the middle of the wake from a spanwise station of approximately $y / b=0.5$ to 0.9 . It is attractive to explain these regions as a result of the streamwise features seen in the oil flow. However, the data presented in this study is not sufficient to resolve these features. The spanwise distributions for sectional lift and drag coefficients for $\alpha=4.27 \mathrm{deg}$. and $\alpha=6.35$ deg. is shown in Fig. 20. These spanwise distributions are much smoother than that of the 3D smooth case, similar to the difference in the streamwise velocity deficit data (Fig. 13 and Fig. 19). There is a discontinuity in these distributions at $y / b=0.55$, which is not seen in other data. It is possible that this discontinuity, along with the regions of high velocity loss seen in the streamwise velocity deficit data, are artifacts created by limitations of the wake survey system, chiefly spatial resolution of the data collected as well as unsteady effects that are unaccounted for in this analysis.

A composite image of the growth of the leading-edge vortices for high-fidelity maximum scallop ice shape for several $\alpha$ is shown in Fig. 21. The first separated vortex forms at $\alpha=6.35 \mathrm{deg}$. and it is similar to that of the 3D smooth shape, except that it extends further toward the wing root. The leading-edge vortex first forms at higher $\alpha$ than both the clean wing and the 3D smooth case. This is due to the existence of valleys between the ice scallop protuberances as shown in Fig. 5b. and Fig. 5c. These gaps create a high-energy, jet-like flow that prevents separation and vortex formation at low-to-moderate alpha. Though these gaps prevent vortex formation and may delay flow separation to higher $\alpha$, there is likely a loss of total pressure associated with flow moving through these gaps. There is some evidence of this in the streamwise velocity deficit diagram in Fig. 19, as these losses would likely cause a thicker wake at lowto-moderate $\alpha$. For $\alpha=7.39$ deg., the leading-edge vortex increases in size, however no additional chord is covered by the vortex. The leading-edge vortex does not extend as far outboard as the vortex at $\alpha=6.35 \mathrm{deg}$. Tip separation also becomes apparent at this $\alpha$, extending from the wing tip to a point on the span near where the leading-edge vortex at $\alpha=6.35 \mathrm{deg}$. swept off the wing. This suggests a complex interaction between the leading-edge vortex and the wing tip vortex. For $\alpha=8.42$ deg., the vortex has extended inboard of the Yehudi break and sweeps off the wing near $y / b=$ 0.45 , much more inboard than the leading-edge vortices at lower $\alpha$. This corresponds well to the mini-tuft imagery for the high-fidelity shape at the same $\alpha$, shown in Fig. 16. In the mini-tuft imagery, separation is seen over the entire chord of the wing for $y / b>0.5$ at $\alpha=8.42 \mathrm{deg}$.

The flowfield associated with the high-fidelity maximum scallop ice shape differs greatly from the flowfield of the clean wing and the 3D smooth maximum scallop ice shape. The existence of the ice shape causes a decrease in lift coefficient, an increase in drag coefficient, and a nose-up pitching moment coefficient compared to the clean case. The high-fidelity case generates larger penalties in lift and in drag below $\alpha=10 \mathrm{deg}$. When comparing changes with $\alpha$, the changes that occur in the high-fidelity maximum scallop ice shape case are more gradual than the clean case. Flow separation on the wing occurs from outboard to inboard as $\alpha$ increases. The gaps that exist in the high-fidelity maximum scallop ice shape cause the aerodynamics to differ from that of the 3D smooth maximum scallop ice shape. These gaps cause streamwise jets to form over the entire wing at low $\alpha$, and in the outboard and inboard regions at moderate $\alpha$. These jets delay leading-edge separation relative to the 3D smooth case. When these jets disappear near the midspan at moderate $\alpha$ and above due to a change in the ice shape geometry, a leading-edge separation region forms. There is some evidence that these jets are manifesting in the wake as small pockets of decreased streamwise velocity perhaps from generated vortices.

Thus, the 3D smooth ice shape is not conservative in the aerodynamic penalties experience by the wing relative to the very complex, high-fidelity case. This is explained by the result of the flow through the valleys or gaps in the ice scallop shape that influence the overall aerodynamics. However, this is one comparison, on one wing geometry, at low Reynolds number and thus extrapolation to other cases is not possible. Future tests are planned to explore this using simple ice shape geometries modelling various gap sizes and spacing at low and moderate Reynolds numbers to better understand this effect. 


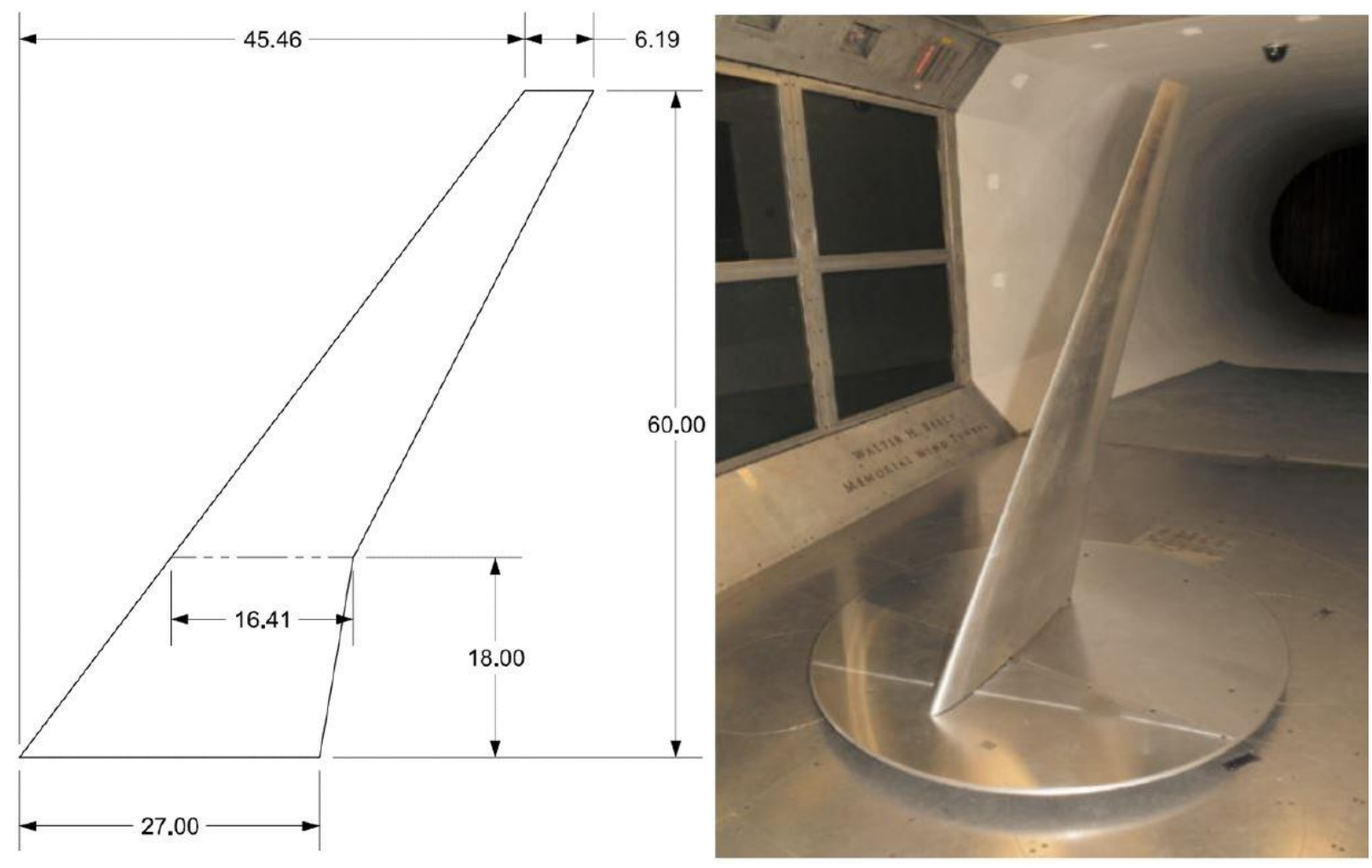

Fig. 1 Summary of $8.9 \%$ scale CRM65 wing model dimensions. 


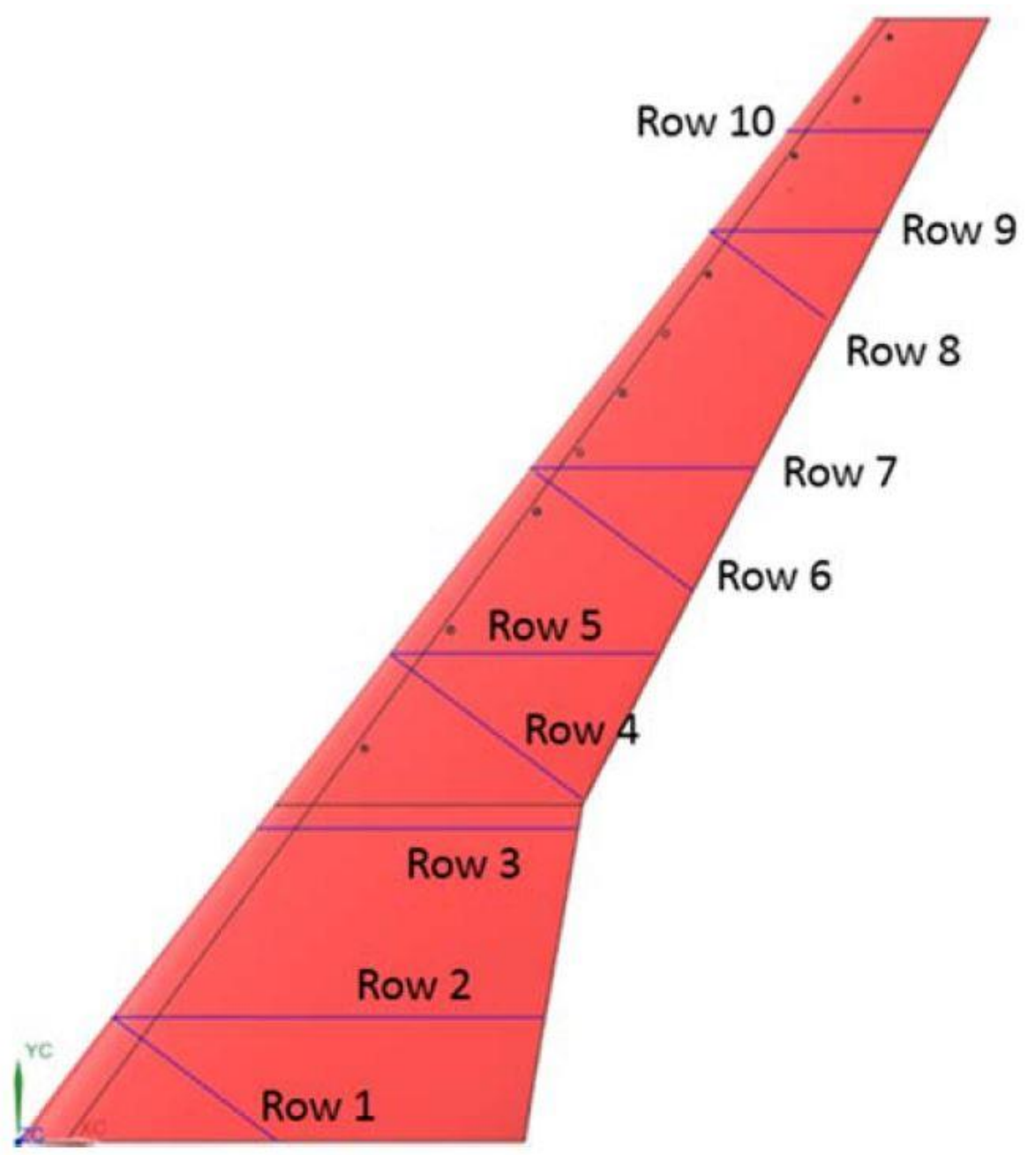

Fig. 2 Location of the pressure tap rows on the upper surface of the wing. 


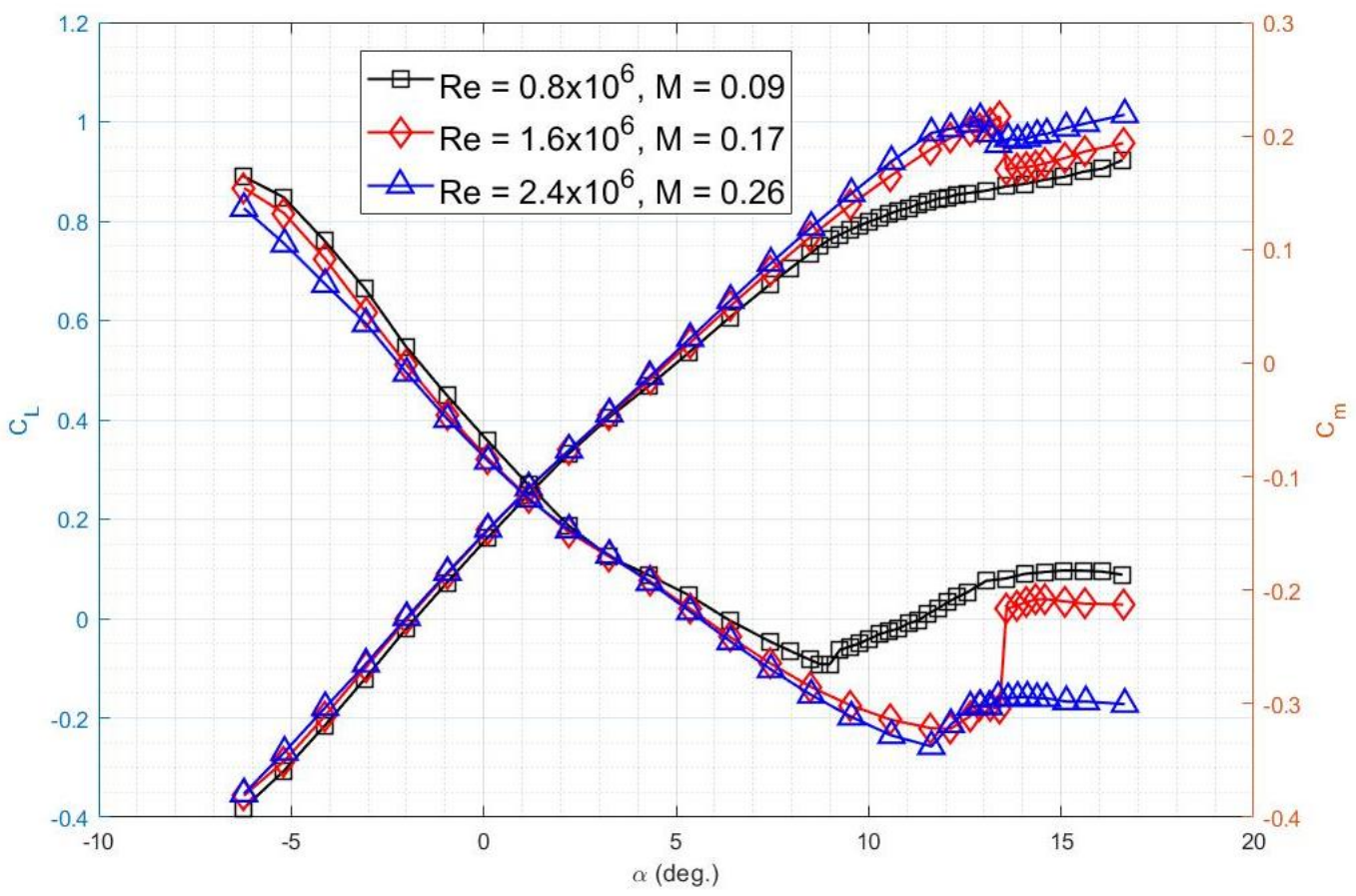

a)

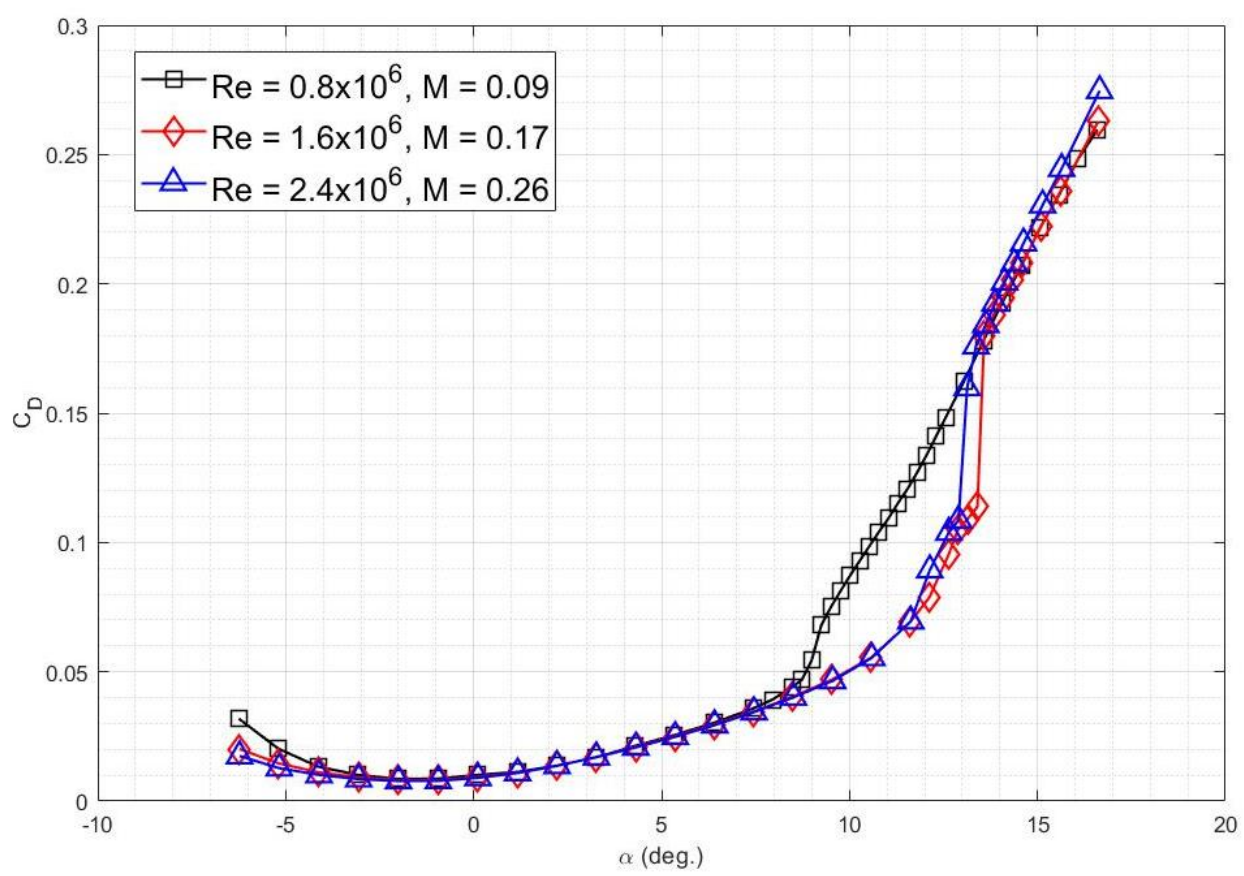

b)

Fig. 3 Aerodynamic performance data is shown for three $R e$ and $M$ combinations for the clean wing. Data presented is: a) $C_{L}$ and $C_{m}$ vs. $\alpha$ and b) $C_{D}$ vs. $\alpha$. 


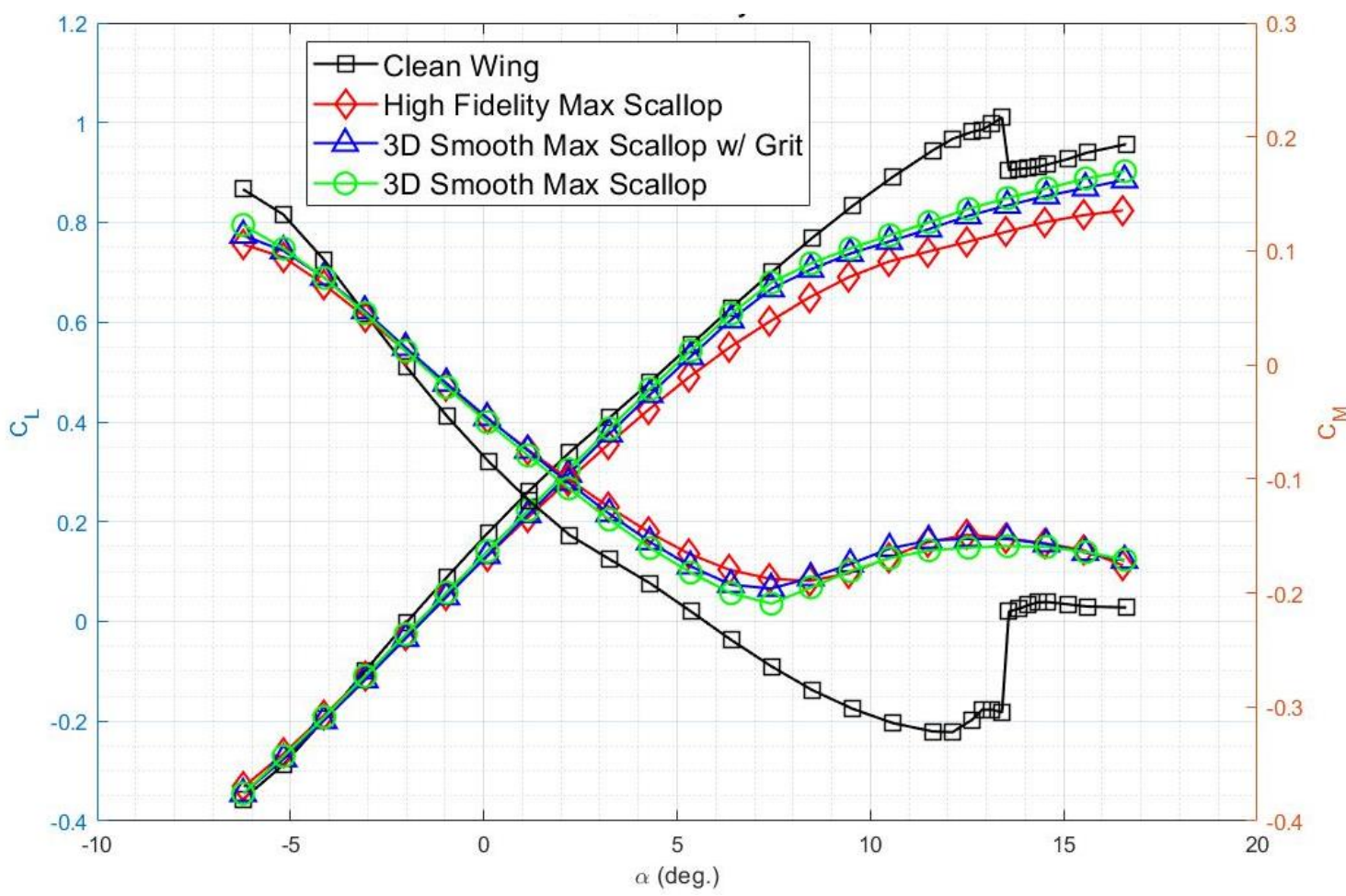

a)

b)

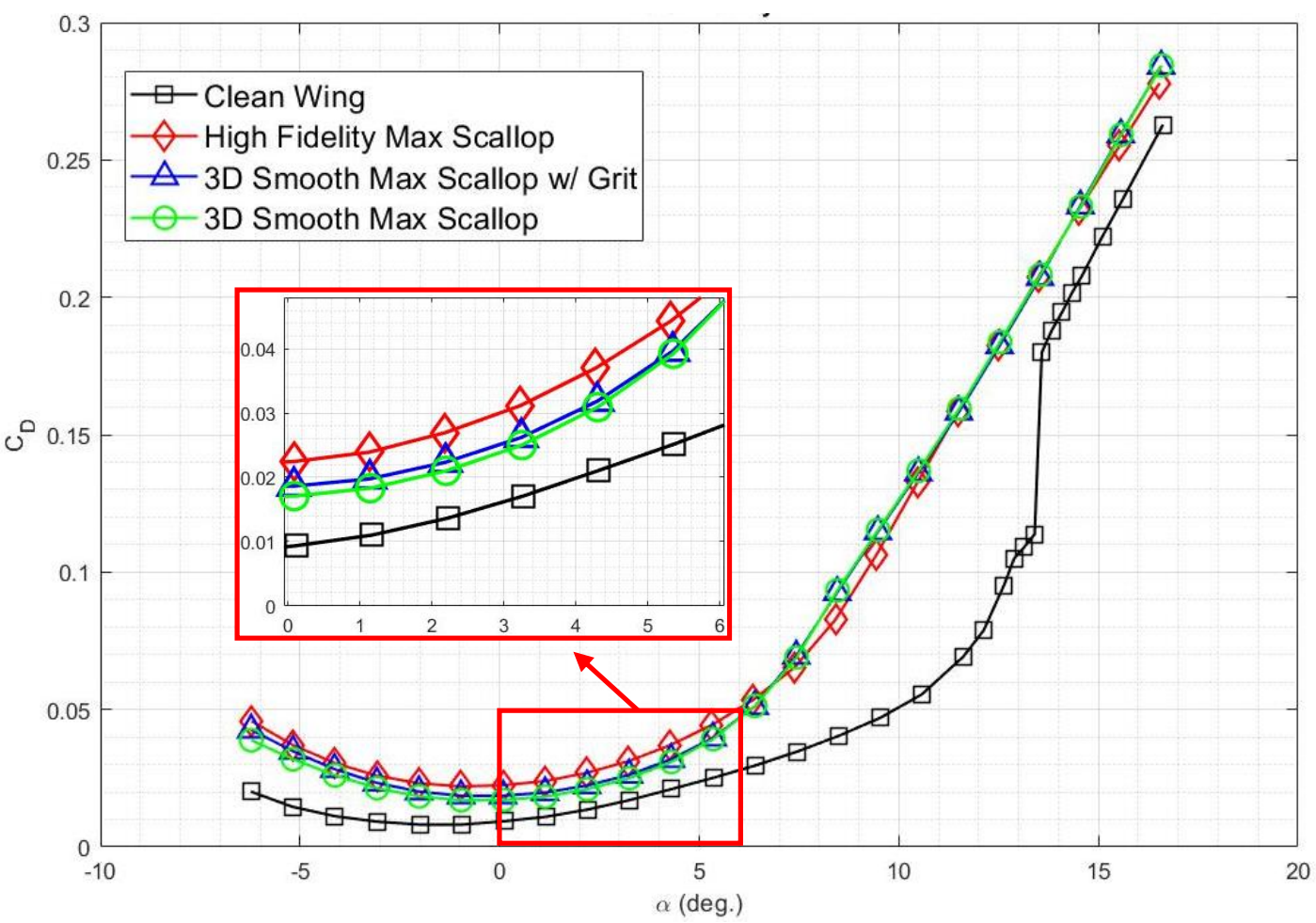

Fig. 4 Aerodynamic performance data is shown for the clean wing and the different fidelities of the maximum scallop ice shape at $\operatorname{Re}=1.6 \times 10^{6}$ and $M=0.17$. Data presented is: a) $C_{L}$ and $C_{m}$ vs. $\alpha$ and b) $C_{D}$ vs. $\alpha$. 


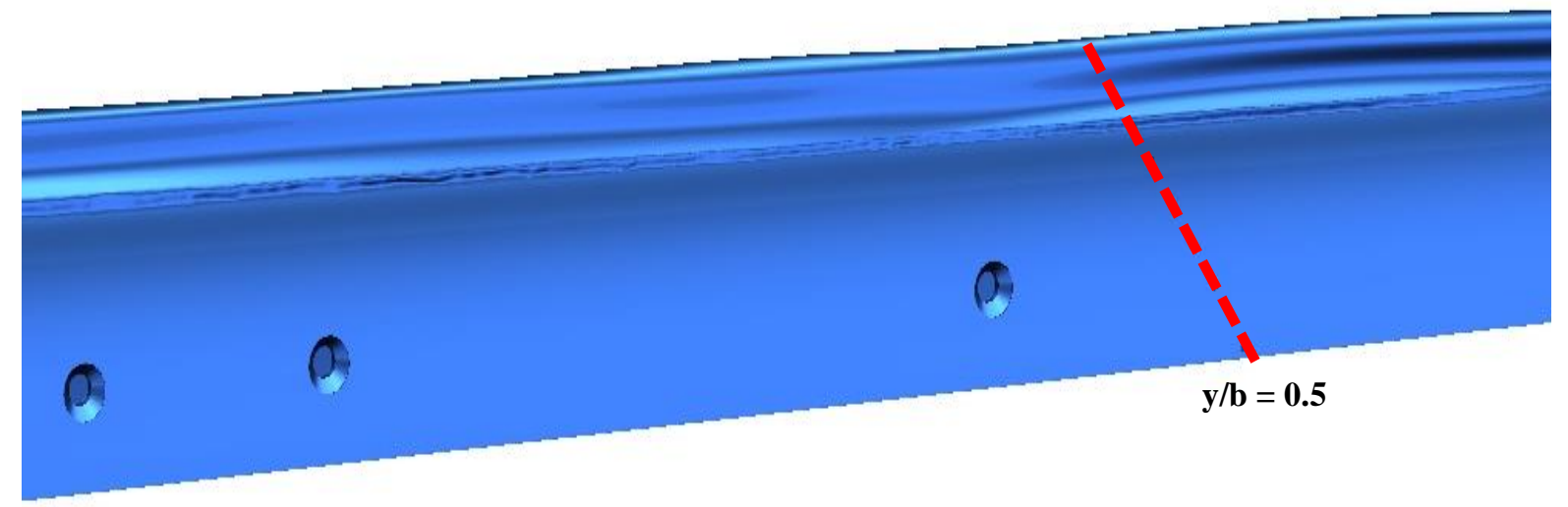

3D Smooth Maximum Scallop

a)

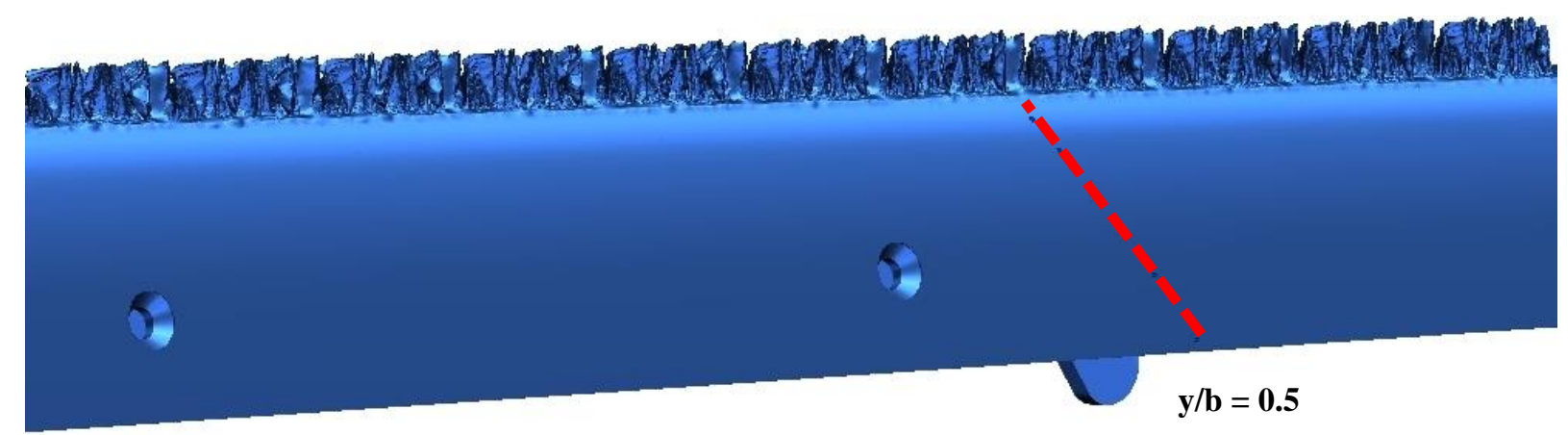

High-Fidelity Maximum Scallop

b)

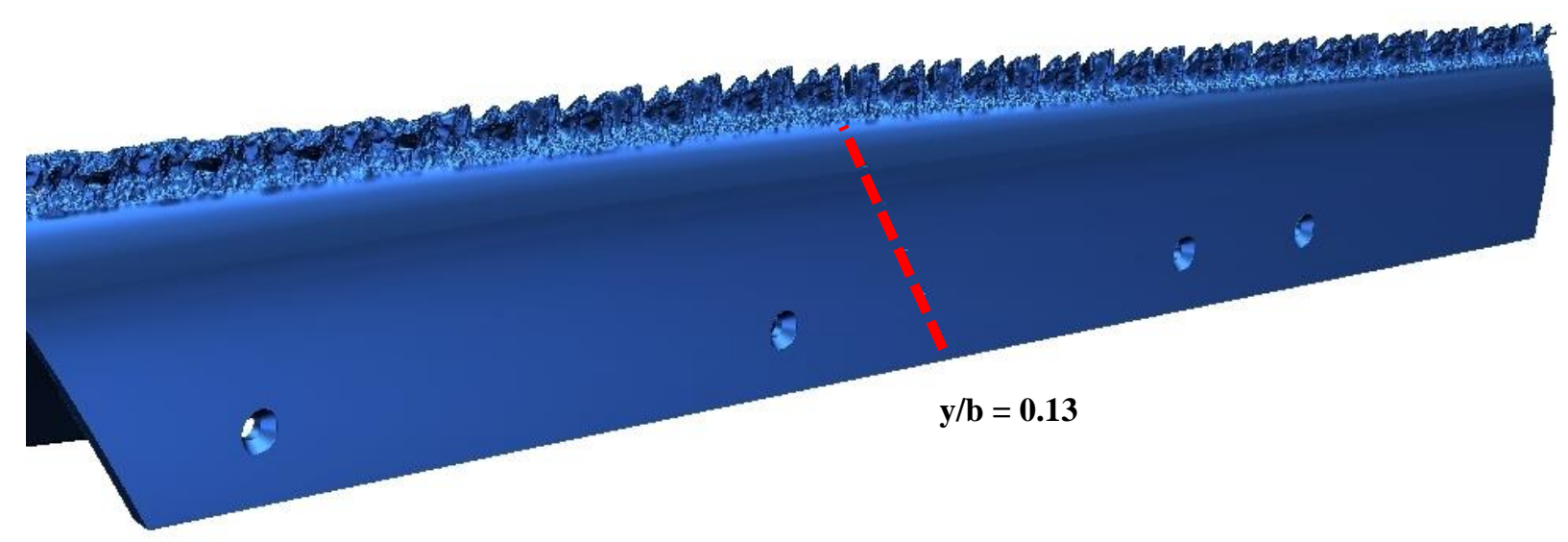

High-Fidelity Maximum Scallop

c)

Fig. 5 Computer-generated render of: a) the 3D smooth maximum scallop ice shape, where the line indicates spanwise station $\mathrm{y} / \mathrm{b}=\mathbf{0 . 5}, \mathrm{b}$ ) the high-fidelity maximum scallop ice shape, where the line indicates spanwise station $y / b=0.5$, and $c$ ) the high-fidelity maximum scallop ice shape, where the line indicates spanwise station $\mathbf{y} / \mathbf{b}=\mathbf{0 . 1 3}$. 


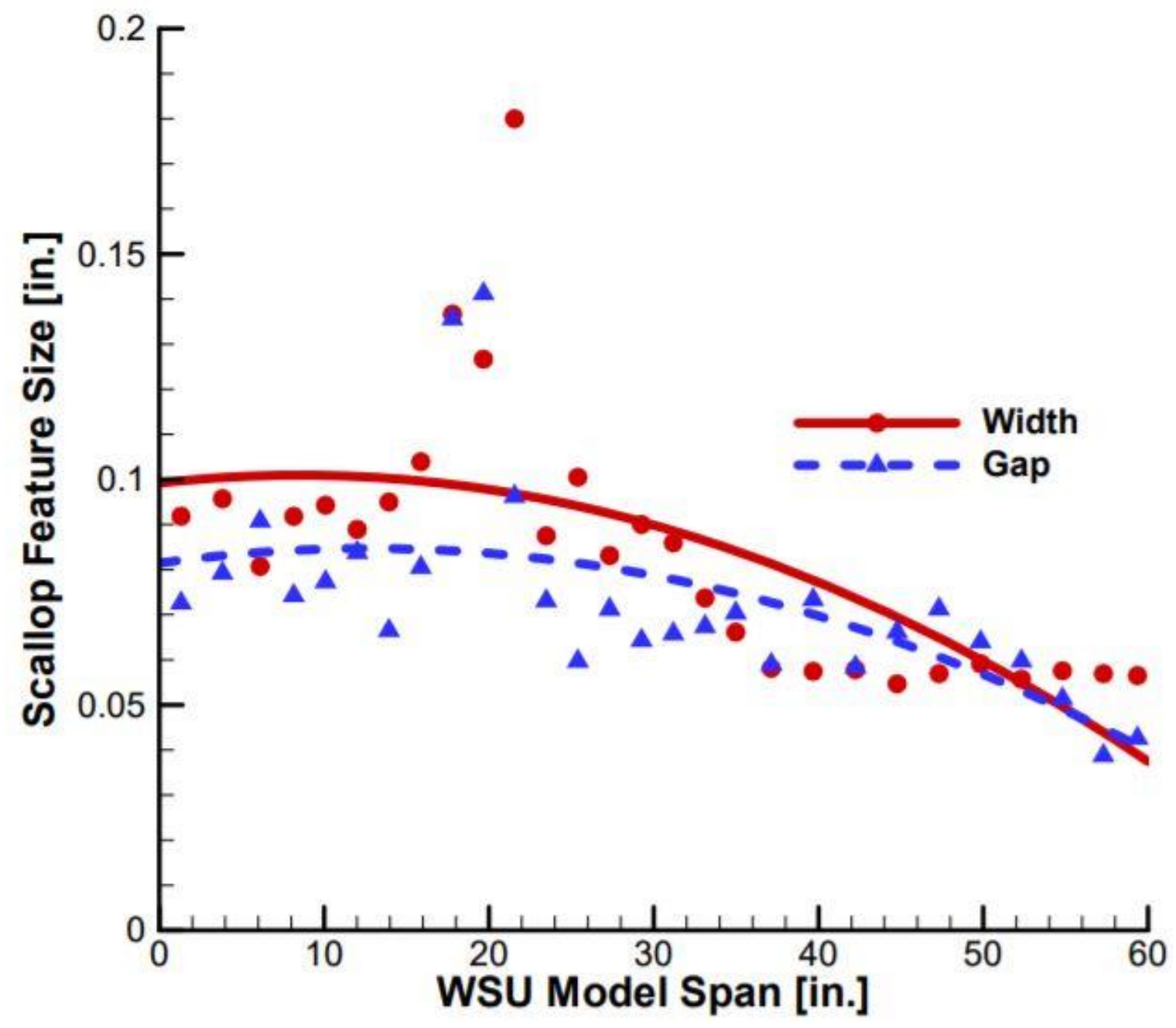

Fig. 6 This plot shows the width of the scallop features and the width of the gaps between the scallop features along with best-fit lines to the data as a function of span for the high-fidelity maximum scallop shape. 


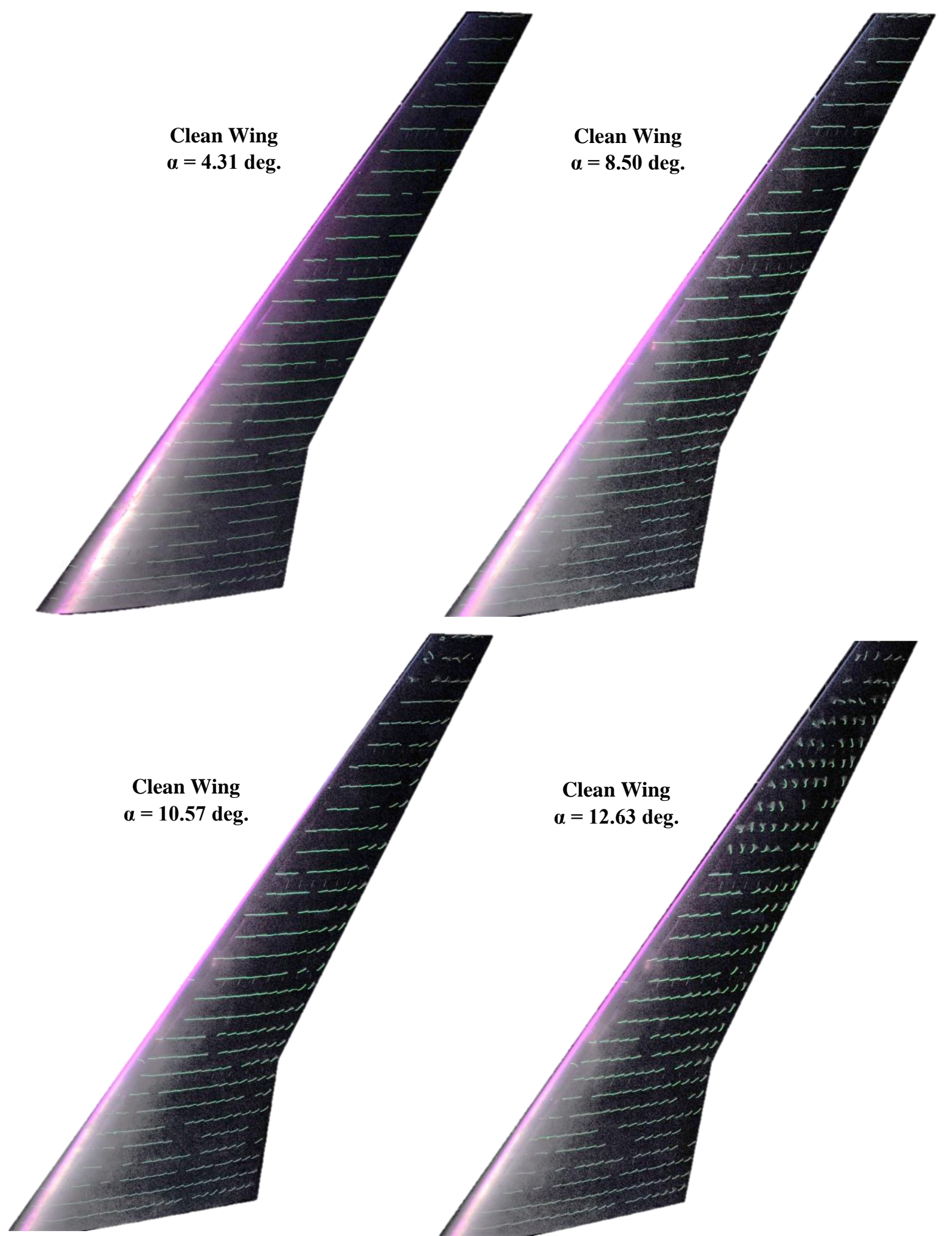

Fig. 7 Mini-tuft imagery is shown for $R e=1.6 \times 10^{6}$ and $M=0.17$ for, top left to bottom right, the clean wing at $\alpha=4.31 \mathrm{deg}$., $8.50 \mathrm{deg}$., $10.57 \mathrm{deg}$., and $12.63 \mathrm{deg}$., respectively. 


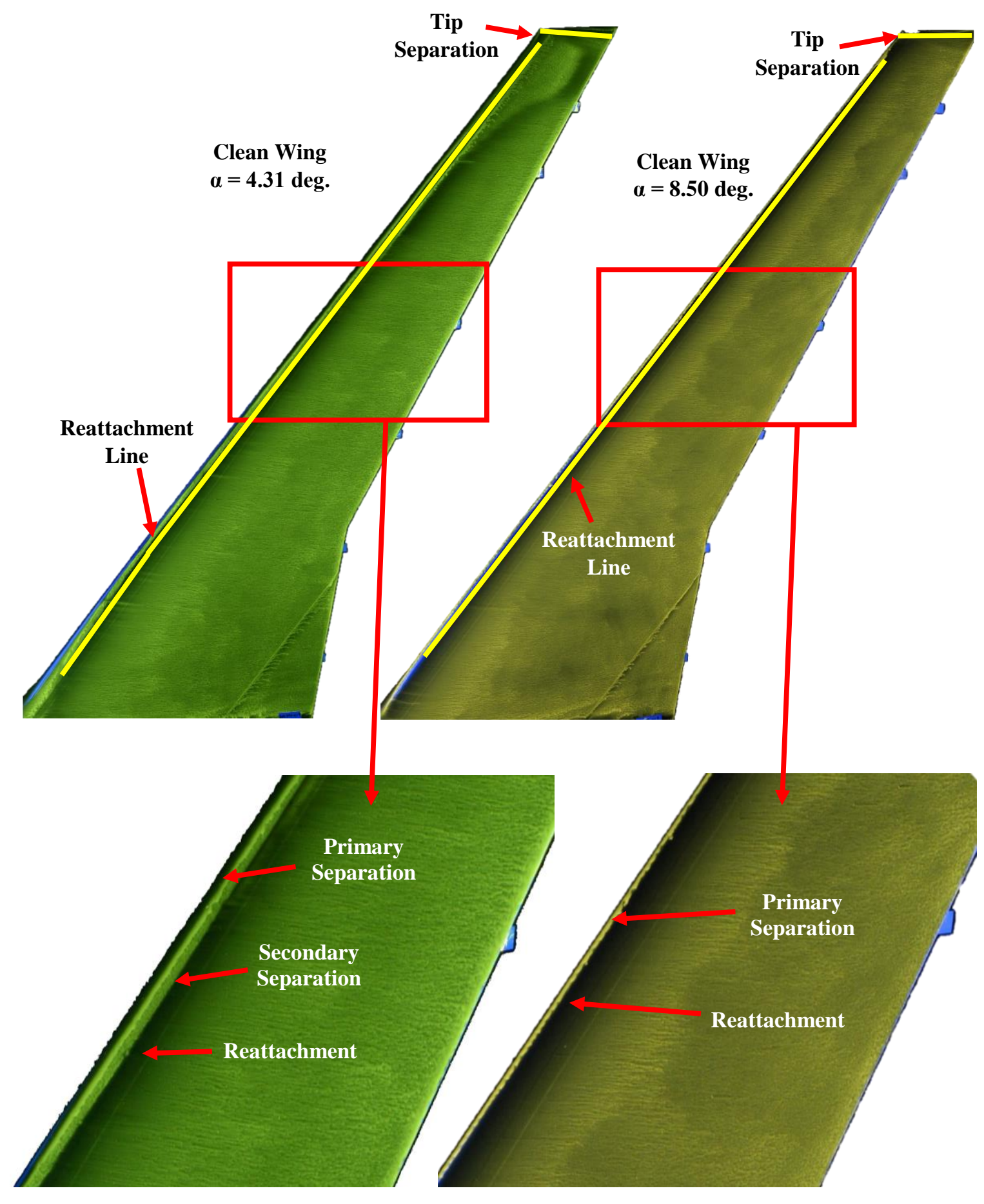

a) 

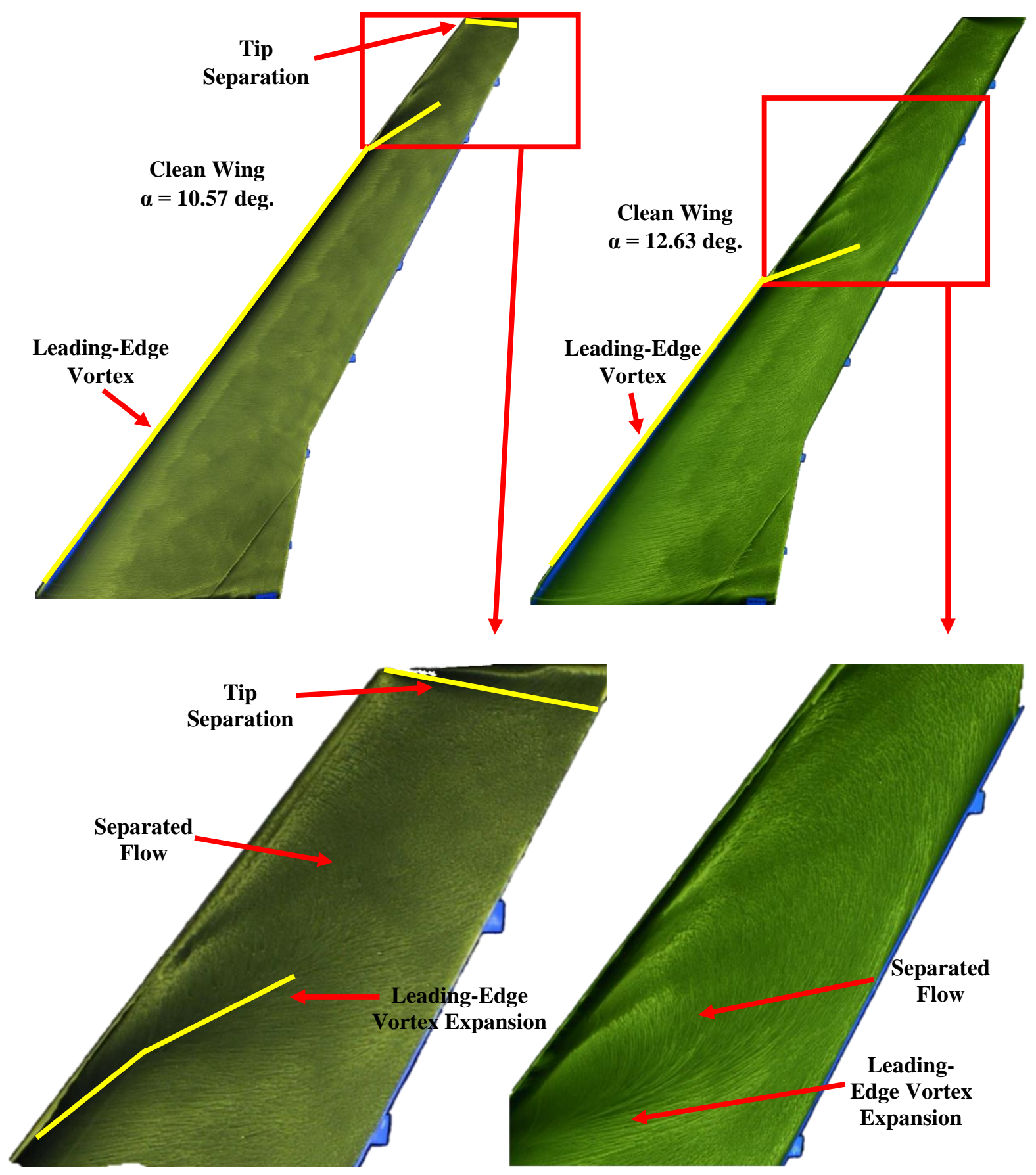

b)

Fig. 8 Oil flow visualization is shown for $R e=1.6 \times 10^{6}$ and $M=0.17$ for the clean wing at a) $\alpha=4.31$ deg. and $8.50 \mathrm{deg}$. and b) $\alpha=10.57 \mathrm{deg}$. and $12.63 \mathrm{deg}$. 

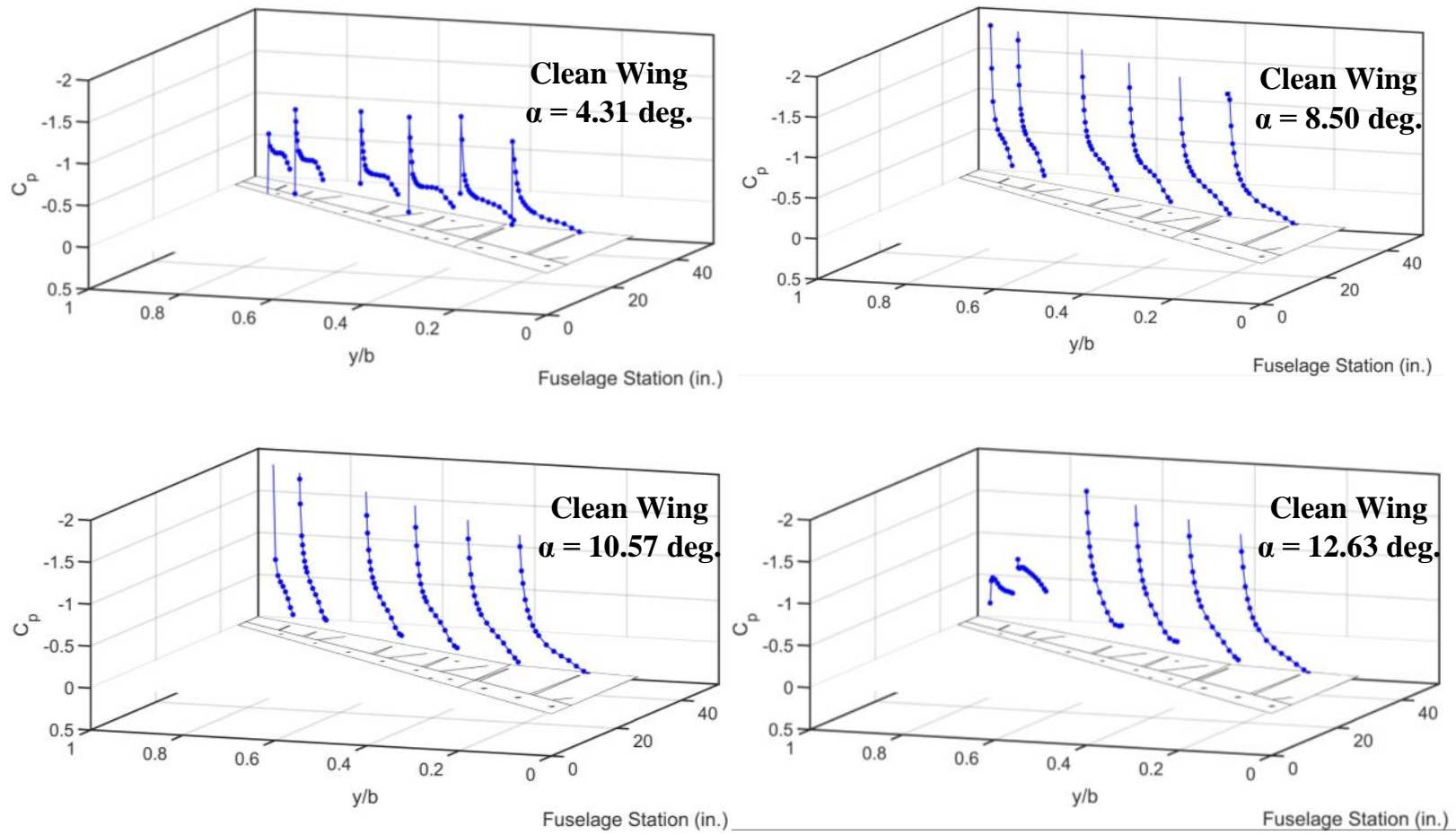

Fig. $9 \mathrm{C}_{\mathrm{p}}$ distribution data is shown for $R e=1.6 \times 10^{6}$ and $M=0.17$ for, top left to bottom right, the upper surface of the clean wing at $\alpha=4.31 \mathrm{deg}$., $8.50 \mathrm{deg}$., $10.57 \mathrm{deg}$., and $12.63 \mathrm{deg}$., respectively. 


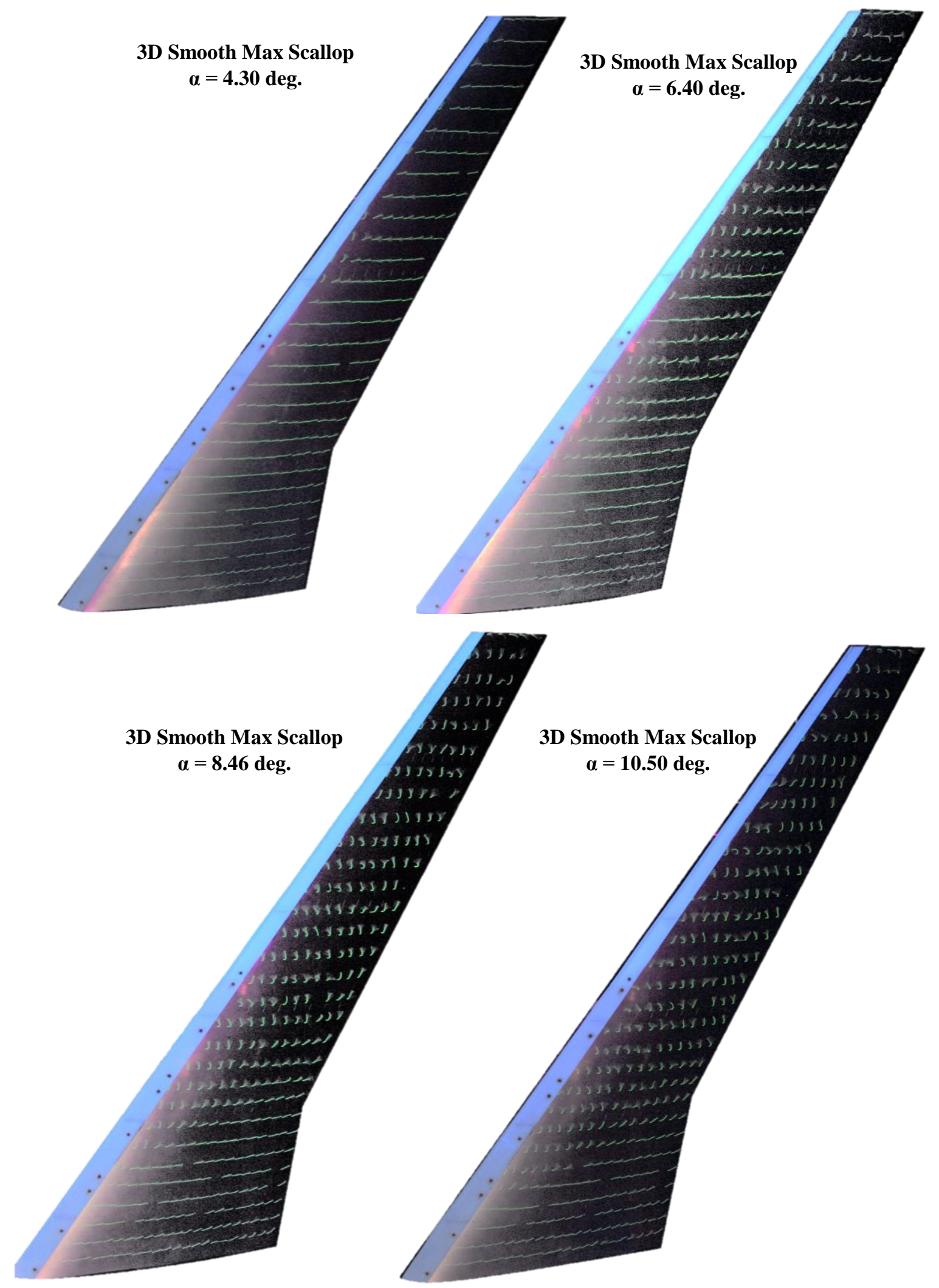

Fig. 10 Mini-tuft imagery is shown for $R e=1.6 \times 10^{6}$ and $M=0.17$ for, top left to bottom right, the 3D smooth maximum scallop ice shape case at $\alpha=4.30$ deg., 6.40 deg., 8.46 deg., and 10.50 deg., respectively. 

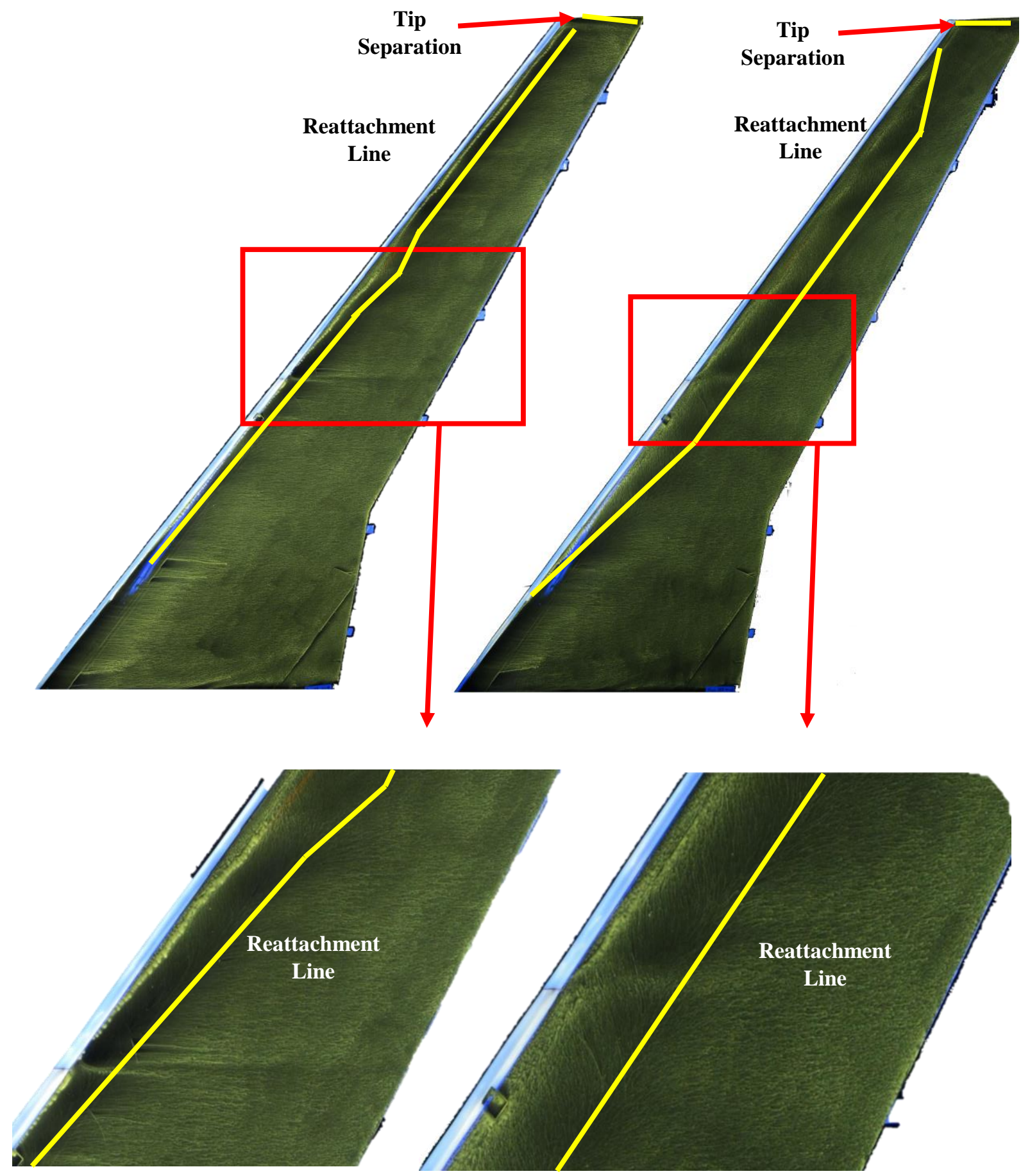

Fig. 11 Oil flow visualization is shown for $R e=1.6 \times 10^{6}$ and $M=0.17$ for the 3D smooth maximum scallop ice shape case at $\alpha=4.30 \mathrm{deg}$. and $\alpha=6.40 \mathrm{deg}$. 

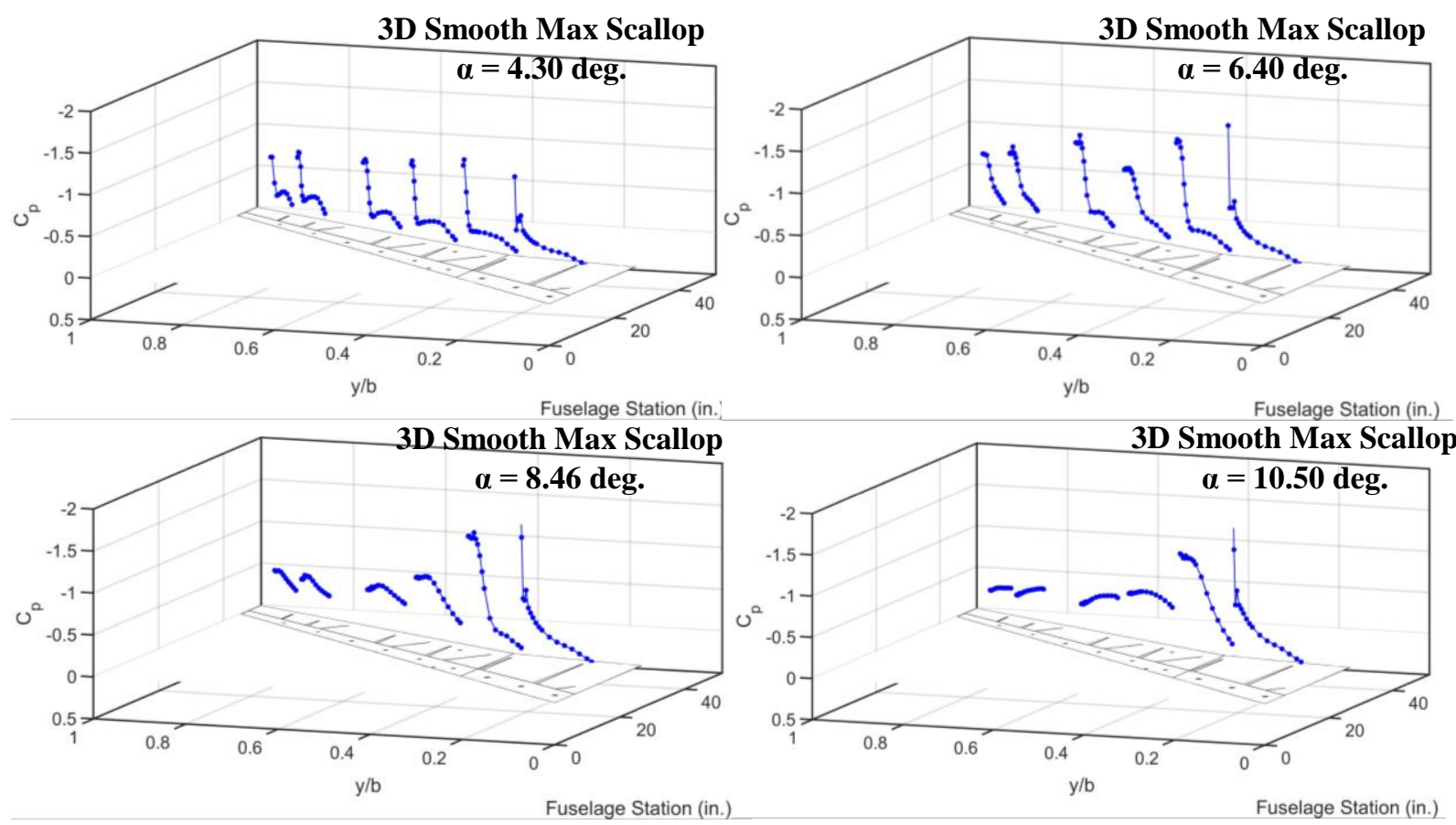

Fig. $12 \mathrm{C}_{\mathrm{p}}$ distribution data is shown for $R e=1.6 \times 10^{6}$ and $M=0.17$ for, top left to bottom right, the upper surface of the 3D smooth maximum scallop ice shape case at $\alpha=4.30 \mathrm{deg}$, $6.40 \mathrm{deg}$., $8.46 \mathrm{deg}$., and $10.50 \mathrm{deg}$., respectively. Note that there are no pressure taps on the ice shape itself. 

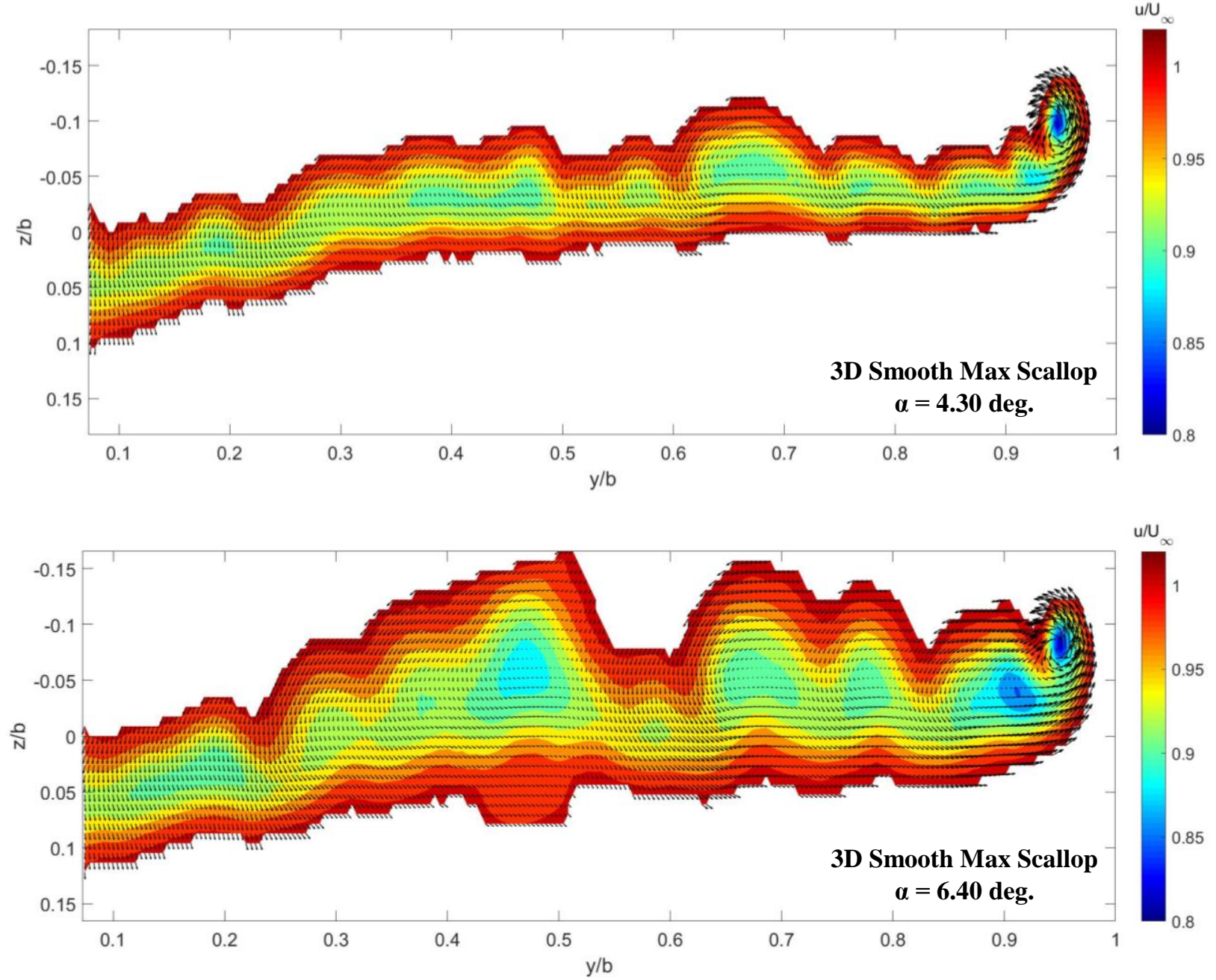

Fig. 13 Streamwise velocity deficit in the wake of the wing is shown for $R e=1.6 \times 10^{6}$ and $M=0.17$ for the 3D smooth maximum scallop ice shape case at $\alpha=4.30 \mathrm{deg}$. and $\alpha=6.40 \mathrm{deg}$. 

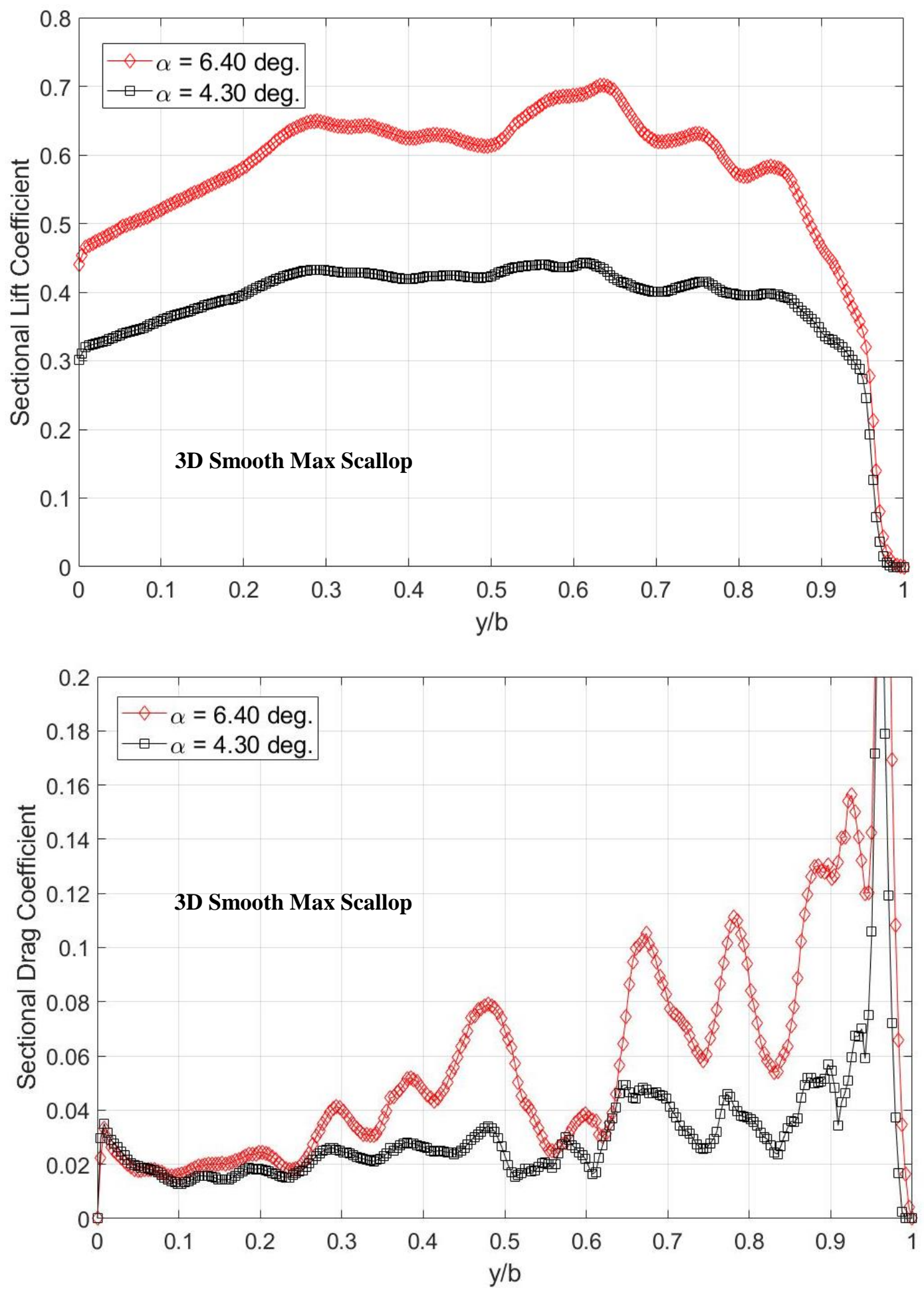

Fig. 14 Spanwise sectional lift coefficient and sectional drag coefficient distributions are shown for $R e=1.6 \mathrm{x}$ $10^{6}$ and $M=0.17$ for the 3D smooth maximum scallop ice shape case at $\alpha=4.30 \mathrm{deg}$. and $\alpha=6.40 \mathrm{deg}$. 


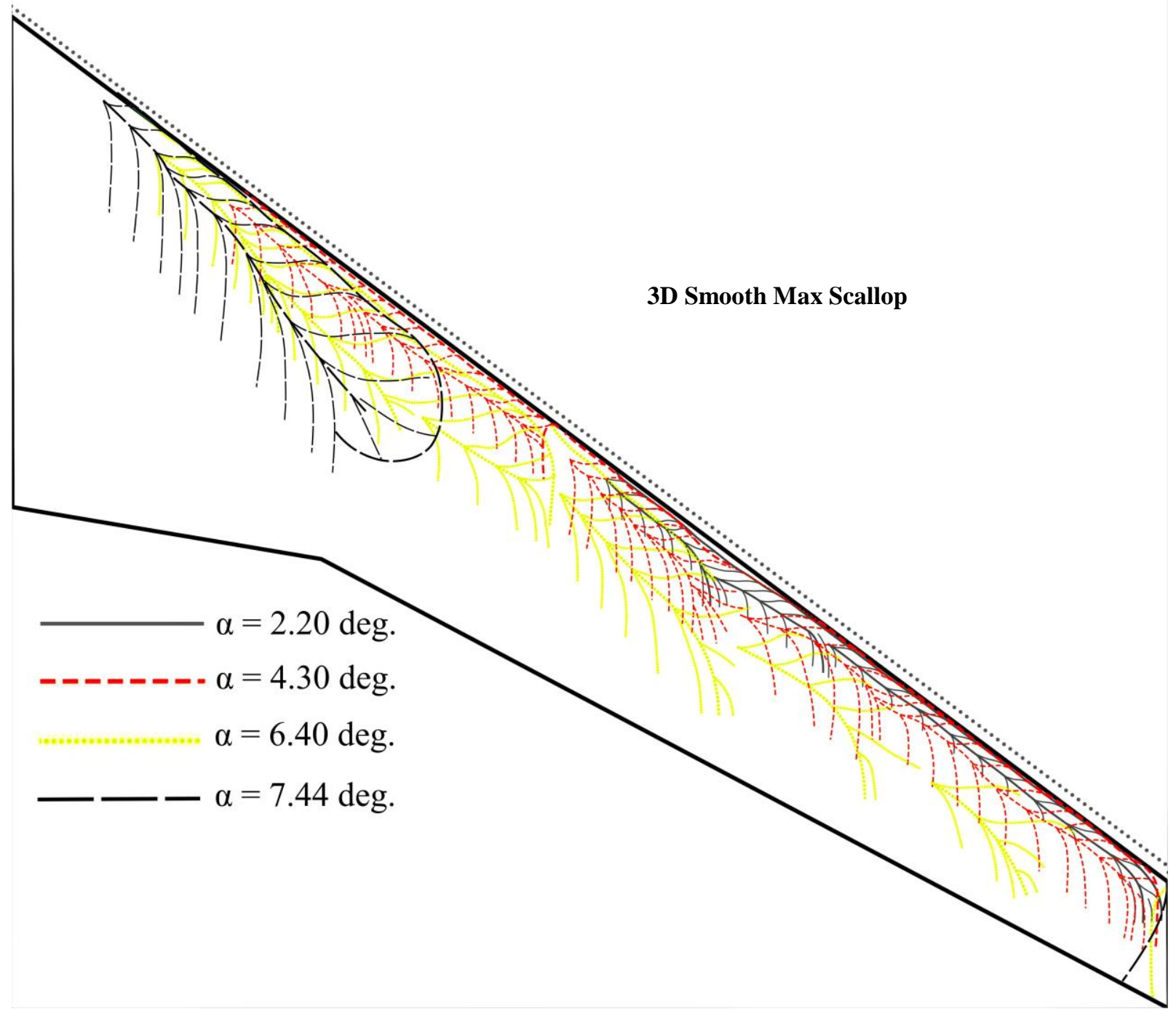

Fig. 15 A composite image of the growth of the leading-edge vortices for $R e=1.6 \times 10^{6}$ and $M=0.17$ for the 3D smooth maximum scallop ice shape for $\alpha=2.20$ deg., $\alpha=4.30$ deg., $\alpha=6.40$ deg., and $\alpha=7.44 \mathrm{deg}$. 


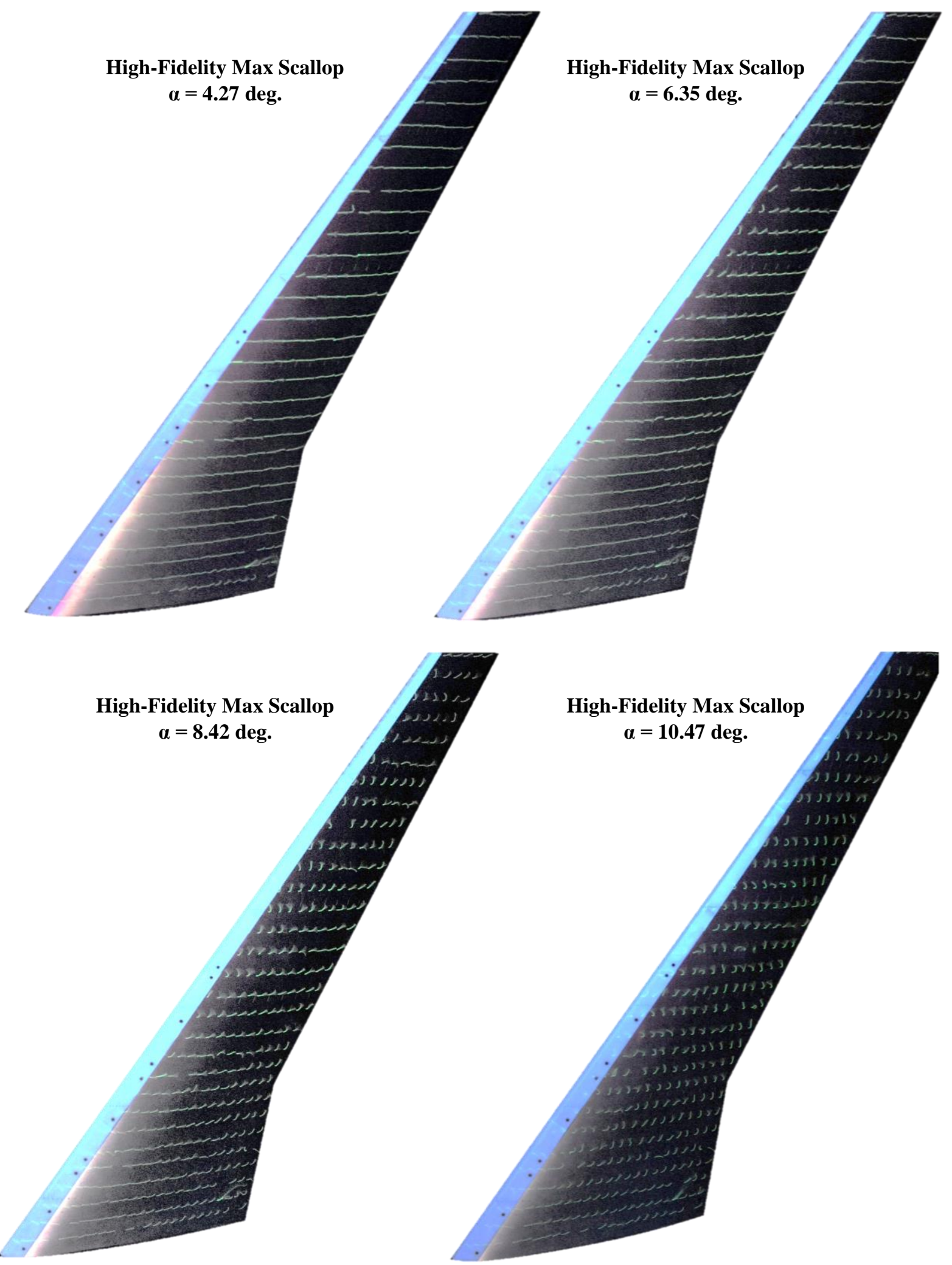

Fig. 16 Mini-tuft imagery is shown for $R e=1.6 \times 10^{6}$ and $M=0.17$ for, top left to bottom right, the highfidelity maximum scallop ice shape case at $\alpha=4.27$ deg., 6.35 deg., $8.42 \mathrm{deg}$., and $10.47 \mathrm{deg}$., respectively. 

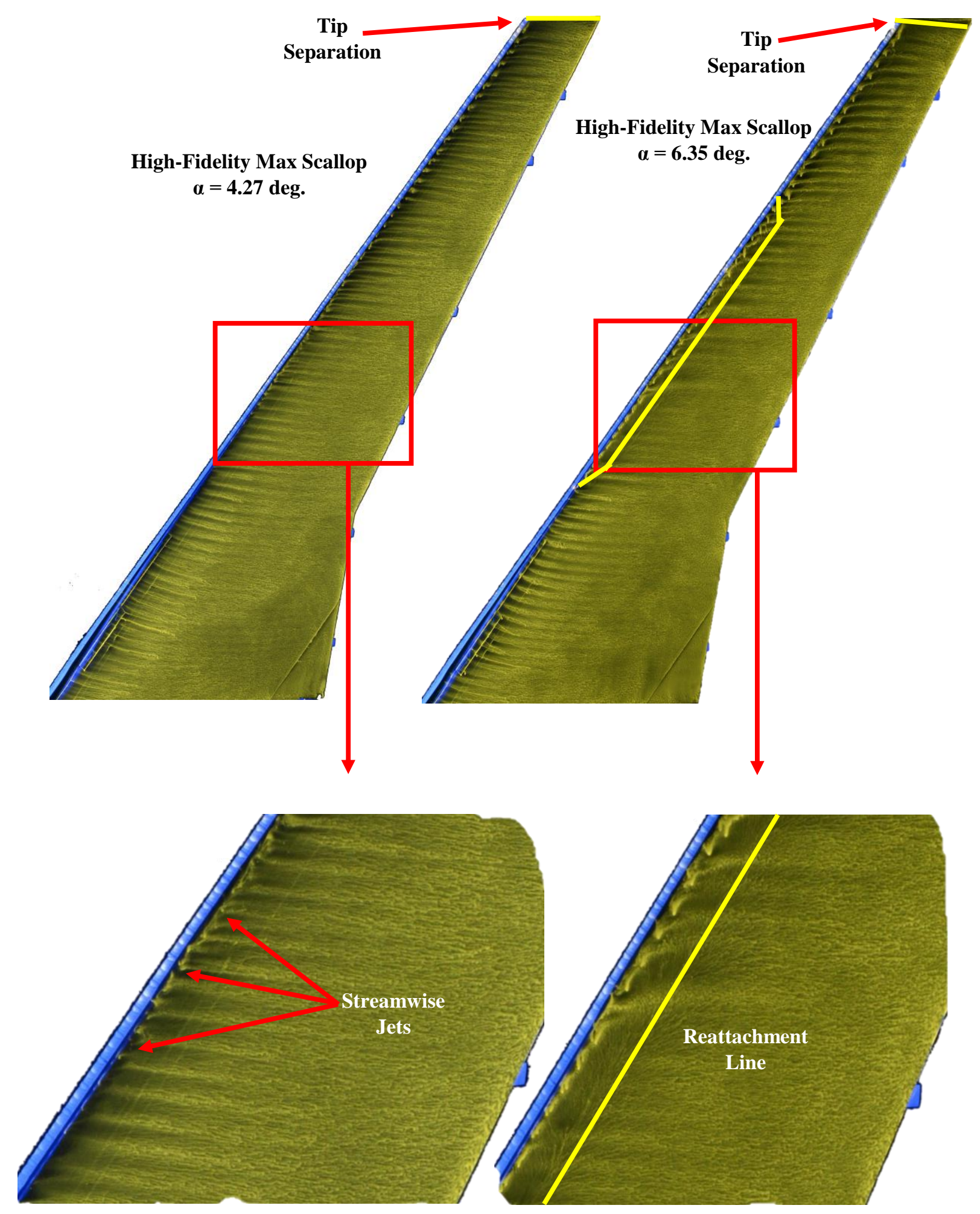

Fig. 17 Oil flow visualization is shown for $R e=1.6 \times 10^{6}$ and $M=0.17$ for the high-fidelity maximum scallop ice shape case at $\alpha=4.27 \mathrm{deg}$. and $\alpha=6.35 \mathrm{deg}$. 

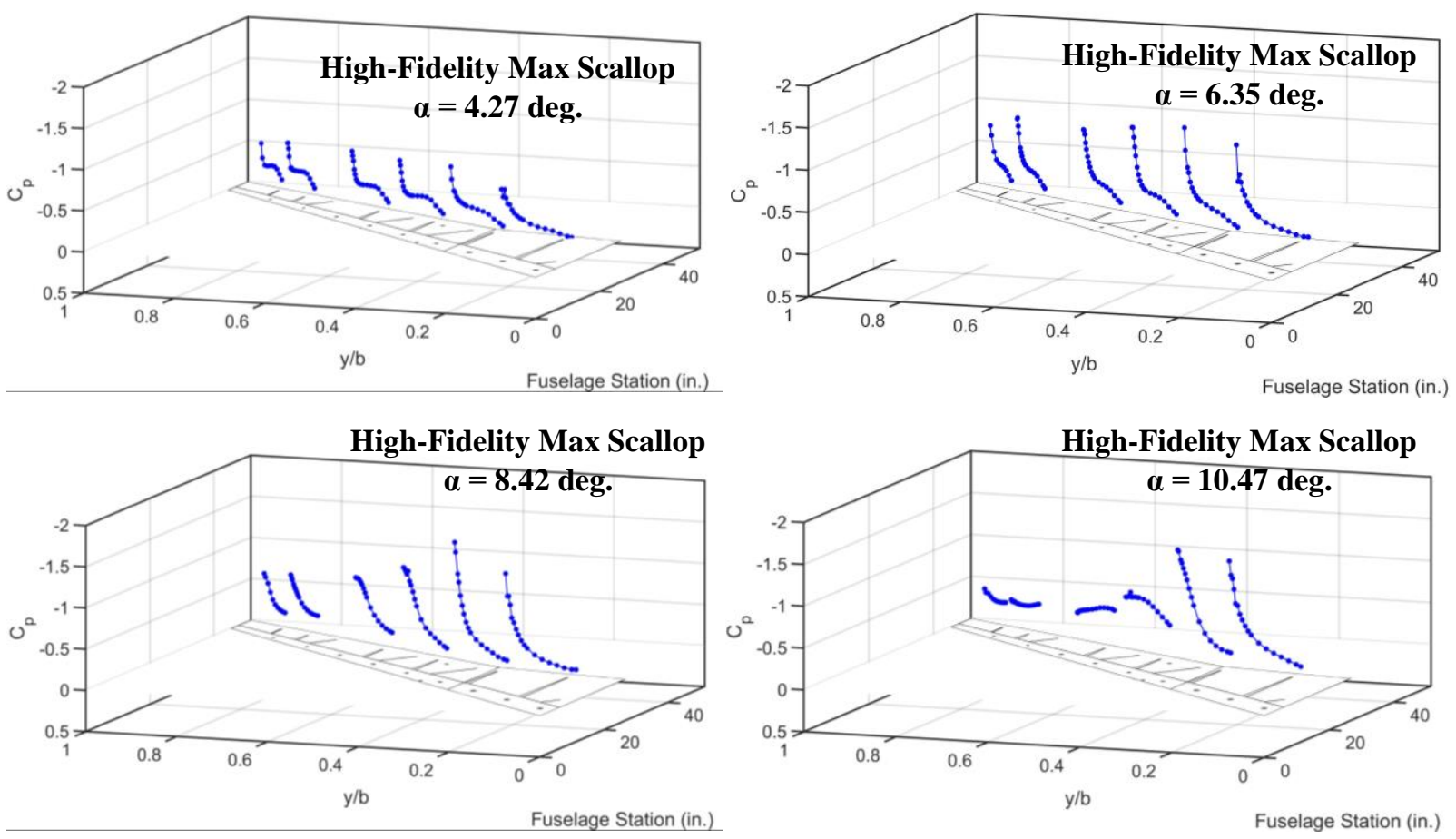

Fig. $18 C_{p}$ distribution data is shown for $R e=1.6 \times 10^{6}$ and $M=0.17$ for, top left to bottom right, the upper surface of the high-fidelity maximum scallop ice shape case at $\alpha=4.27 \mathrm{deg}$., $6.35 \mathrm{deg}$., $8.42 \mathrm{deg}$., and 10.47 deg., respectively. Note that there are no pressure taps on the ice shape itself. 

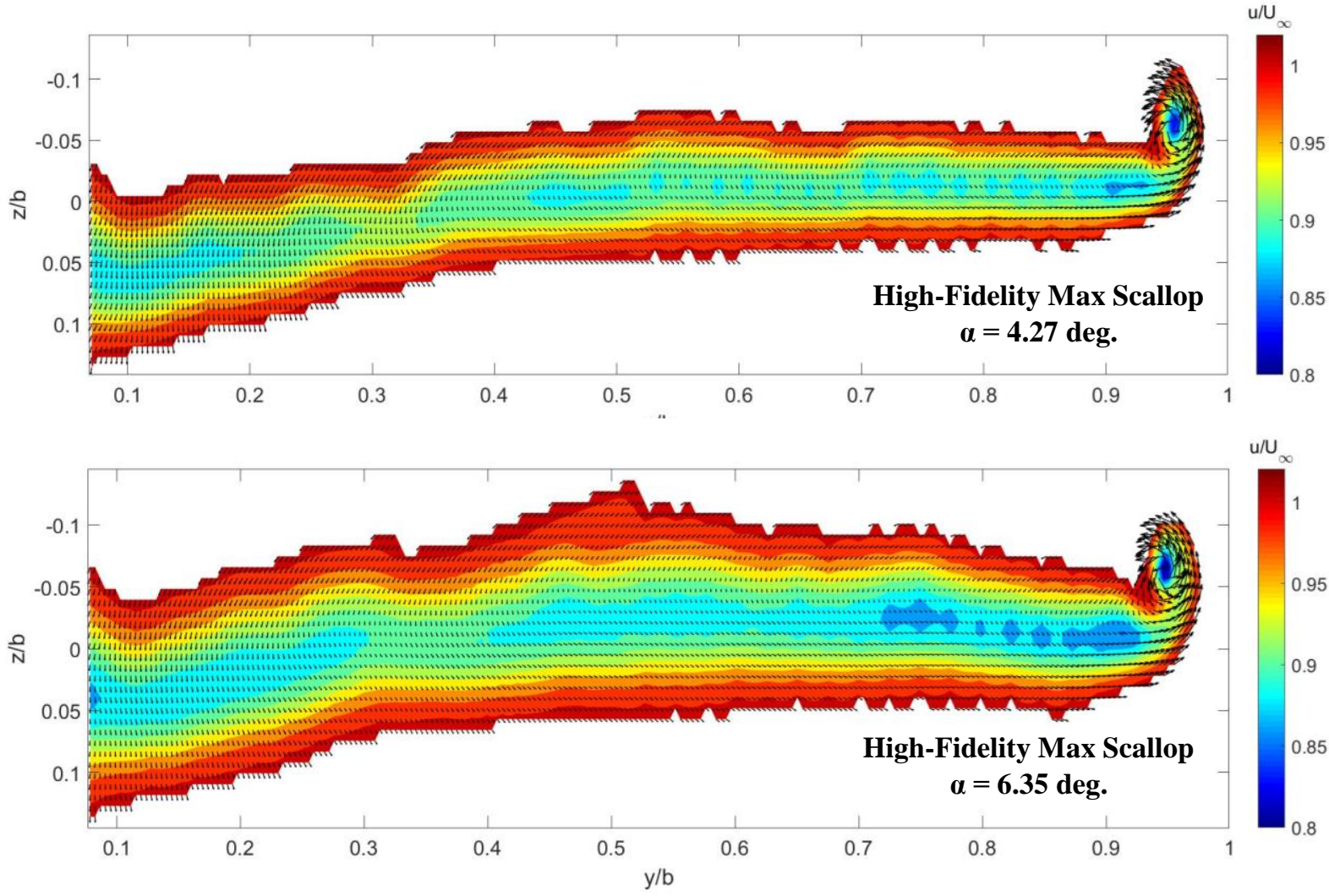

Fig. 19 Streamwise velocity deficit in the wake of the wing is shown for $R e=1.6 \times 10^{6}$ and $M=0.17$ for the high-fidelity maximum scallop ice shape case at $\alpha=4.27 \mathrm{deg}$. and $\alpha=6.35 \mathrm{deg}$. 

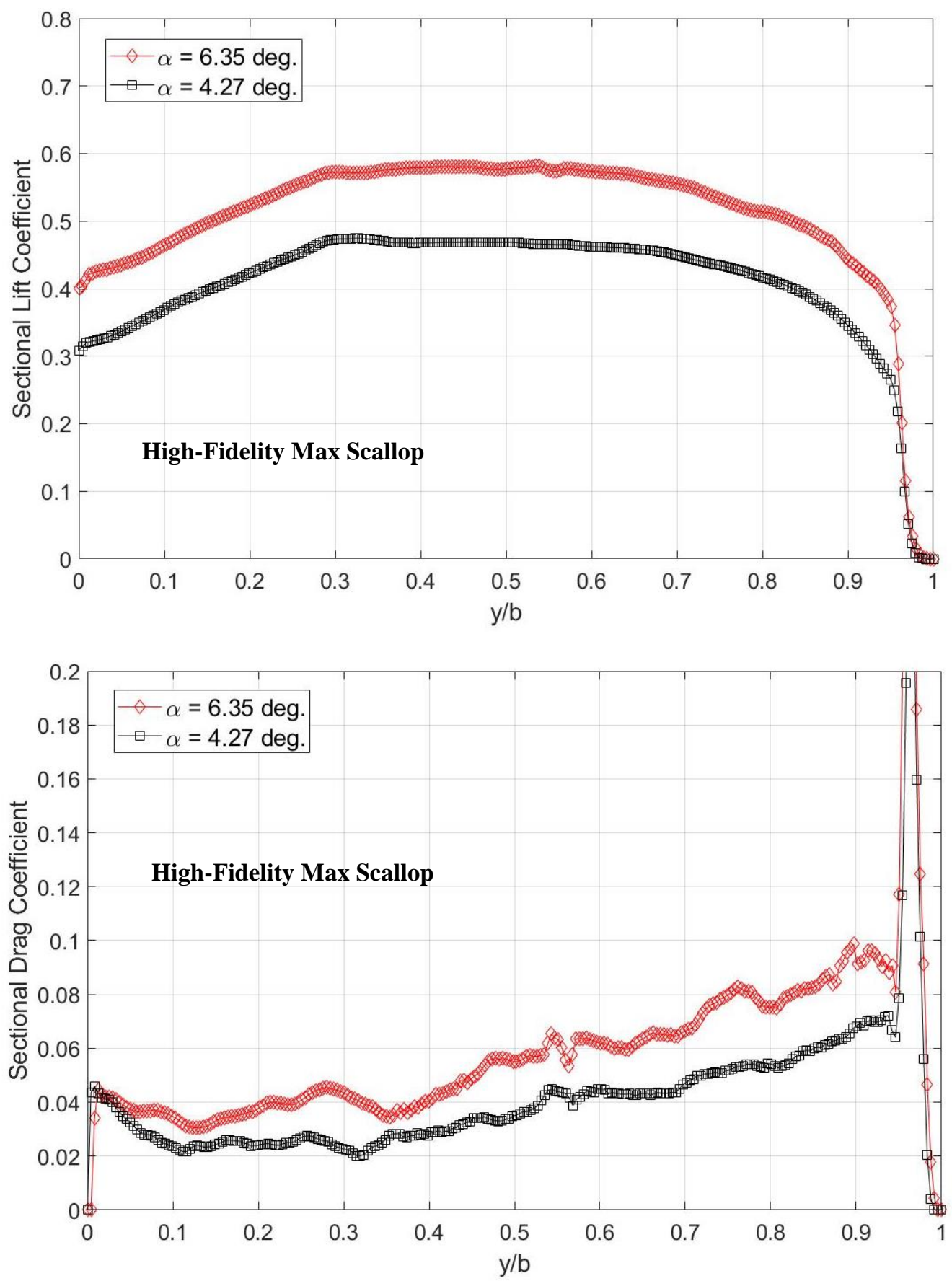

Fig. 20 Spanwise sectional lift coefficient and sectional drag coefficient distributions are shown for $R e=1.6 \mathrm{x}$ $10^{6}$ and $M=0.17$ for the high-fidelity maximum scallop ice shape case at $\alpha=4.27 \mathrm{deg}$. and $\alpha=6.35 \mathrm{deg}$. 


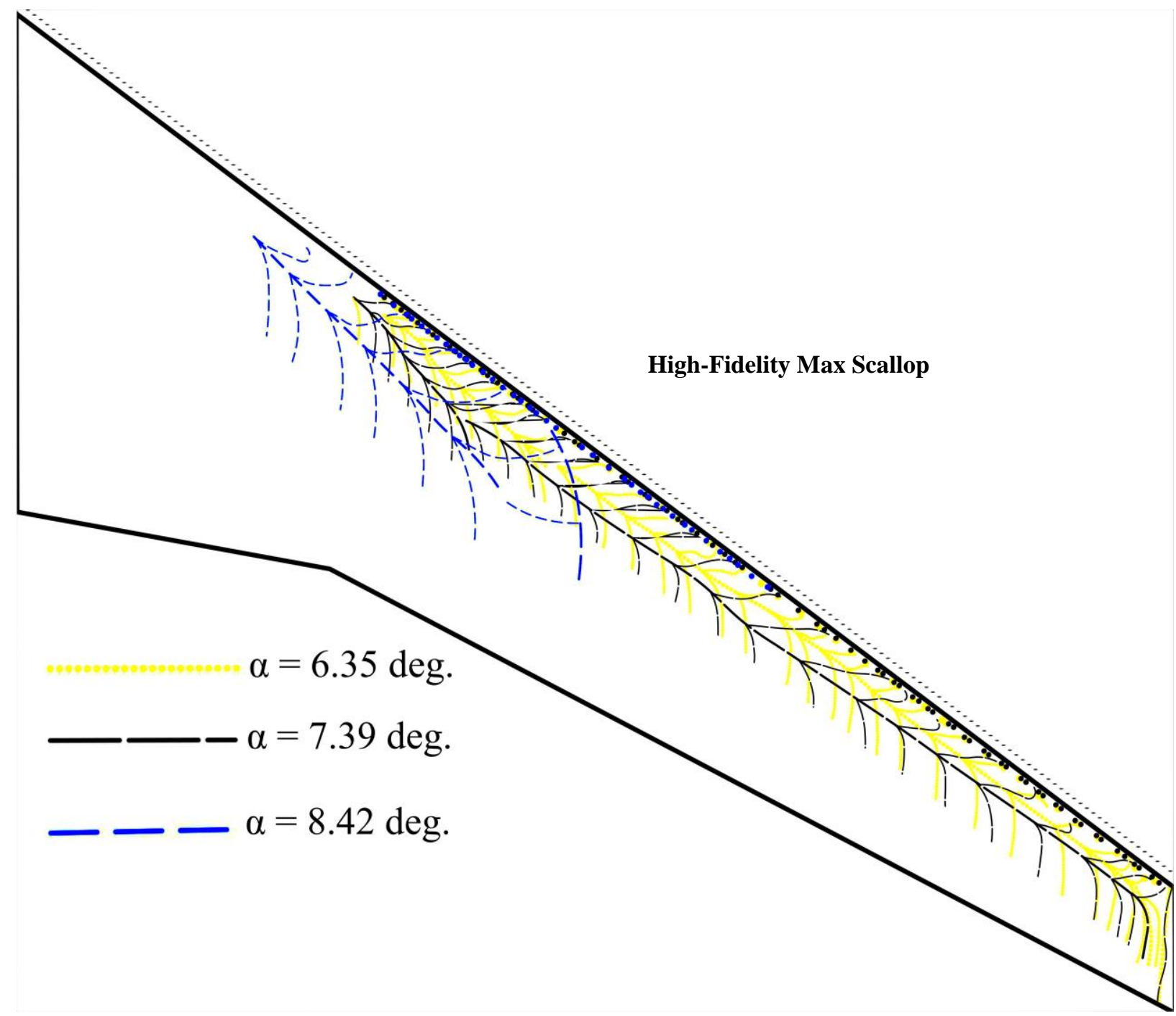

Fig. 21 A composite image of the growth of the leading-edge vortices for $R e=1.6 \times 10^{6}$ and $M=0.17$ for the high-fidelity maximum scallop ice shape for $\alpha=6.35 \mathrm{deg} ., \alpha=7.39 \mathrm{deg}$. , and $\alpha=8.42 \mathrm{deg}$.

\section{Conclusions and Recommendations}

This paper examined the flow visualization images, wake measurements, and balance data for a clean swept-wing, as well as the same wing with two fidelities of a leading-edge ice shape. Data analysis presented in this paper aims to bridge the understanding between the numerical performance data such as variation of $C_{L}$ and $C_{D}$ with $\alpha$ and the flow phenomena occurring due to ice on the leading edge of a swept wing. The results lead to several conclusions:

1. The clean wing experienced an aggressive, tip-first stall, which was not seen for the wing with the different fidelities of the maximum scallop ice shape.

2. The clean wing had a small, relatively strong leading-edge vortex at lower $\alpha$, while the iced cases tend to have much larger, seemingly weaker leading-edge vortices. The size of the leading-edge vortex varies greatly between the two fidelities of the maximum scallop ice shape. 
3. The high-fidelity shape generated streamwise flow features that repeat spanwise downstream of the ice simulation generated from flow through the gaps in the shape. These "jets" are important to the overall aerodynamics of the wing.

4. The 3D smooth maximum scallop ice shape tested was non-conservative in its aerodynamic penalties as compared to the full high-fidelity case.

5. The variation in the scallop shape resulting from the morphing process used to generate it is seen in the aerodynamics near the midspan of the wing, where the ratio of scallop width to gap width in the shape is increased. This variation, which is more extreme than the variation in the $3 \mathrm{D}$ smooth shape, effects the formation of the leading-edge vortex by preventing the streamwise flow features from forming over the highfidelity shape.

These results also lead to recommendations for this work:

1. More information is need to more fully understand the effect of scallop ice shape gaps on aerodynamics and before these data can be extrapolated to a more general result. Artificial ice geometry wind tunnel tests that varies the gap size and spacing are planned over a range of $R e$ and $M$ combinations to improve our understanding of the phenomena observed in these tests.

2. Improved measurements and visualization could improve our understanding of the streamwise features seen in the high-fidelity case and how it impacts the flowfield and resulting iced swept-wing aerodynamics.

a. Off-body flow visualization could be utilized to observe more closely the jet features and their interaction with and effect on the overall flow.

b. Pressure taps on the ice shape itself such that the pressure coefficient on the leading edge of the wing can be measured with an ice shape attached would be very useful.

c. More spatial fidelity in the wake survey technique would aid in understanding the smaller features in the flow, such as the potential vortices that manifest at the wake plane. This would yield a better understanding of the flowfield over the wing itself. This could be coupled with performing wake survey measurements at multiple streamwise planes, allowing for a study of the evolution of the wake and flowfield.

\section{Appendix}

The uncertainty of the measured data is provided in this appendix. This uncertainty analysis, as well as associated hardware, is described in more detail by Broeren et al [10]. The results of that analysis are summarized here for convenience. Load measurements in the Walter H. Beech Wind Tunnel at Wichita State University were taken using a 6-component, pyramidal-style force balance located beneath the test section floor. The accuracy of the balance was $0.02 \%$ of full-scale. The uncertainty in the measured dynamic pressure was approximately $\pm 0.1 \mathrm{psf}$. Two models of pressure tap were used for collecting surface pressure data. Near the leading edge, miniature electronic pressure scanning modules developed by Esterline were used (model ESP-32HD). These modules had a range of $\pm 10.0 \mathrm{psi}$. The remaining pressure taps used modules with a range of \pm 2.0 psi. The accuracy of the modules was $\pm 0.03 \%$ and $\pm 0.06 \%$ for the $\pm 10.0 \mathrm{psi}$ and $\pm 2.0 \mathrm{psi}$ modules, respectively. The uncertainty of the modules is $\pm 0.003 \mathrm{psi}$ for the \pm 10.0 psi module and \pm 0.0012 psi for the \pm 2.0 psi module.

Uncertainties in experimental data for an example data point ( $\alpha=4$ deg., $R e=2.4 \times 10^{6}$, and $M=0.27$ ) are given in Table 3, courtesy of Broeren et al [10]. The root-sum-square method outlined by Coleman and Steele [21] and developed by Kline and McClintock [22] was used to determine these uncertainties.

Table 3. Absolute and relative uncertainties for example data point for $R e=2.4 \times 10^{6}$ and $M=0.27$.

\begin{tabular}{|c|c|c|c|}
\hline Variable & Reference Value & Absolute Uncertainty & Relative Uncertainty \\
\hline$\alpha$ & $4.00 \mathrm{deg}$. & $\pm 0.05 \mathrm{deg}$. & $\pm 1.25 \%$ \\
\hline$C_{L}$ & 0.5029 & \pm 0.00137 & $\pm 0.27 \%$ \\
\hline$C_{D}$ & 0.0215 & \pm 0.00068 & $\pm 3.15 \%$ \\
\hline$C_{m}$ & -0.0067 & \pm 0.0006 & $\pm 9.01 \%$ \\
\hline
\end{tabular}




\section{Acknowledgments}

The authors would like to thank Dr. Mark Potapczuk from NASA for his contributions to the test preparation and execution. The team would also like to thank the many members at the University of Washington Aircraft Icing and Aerodynamics Research Group including Stephanie Camello, Gustavo Fujiwara, and Kevin Ho for their contributions to test preparation, execution, and analysis. John Laffen, Kevin Kelly, Aaron Kuen, and Ryan Benyshek at the Walter H. Beech Memorial Wind Tunnel at Wichita State University were immensely helpful with test preparation, execution, and data acquisition. The authors would also like to thank Dr. James Riley and Timothy Smith from the FAA for their contributions to the project. This work was funded by FAA grant 15-G-009.

\section{References}

[1] Bragg, M. B., Broeren, A. P., and Blumenthal, L. A., "Iced-Airfoil Aerodynamics," Progress in Aerospace Sciences, Vol. 41, No. 5, 2005, pp. 323-362

[2] Broeren, A. P., Potapczuk, M. G., Riley, J. T., Villedieu, P., Moens, F., and Bragg, M. B., "Swept-Wing Ice Accretion Characterization and Aerodynamics," Proceedings of the 5th AIAA Atmospheric and Space Environments Conference, AIAA Paper 2013-2824, San Diego, CA, June 2013.

[3] Rivers, M. and Dittberner, A., "Experimental Investigation of the NASA Common Research Model," AIAA Paper 2010-4218, June 2010.

[4] Broeren, A.P., Potapczuk, M.G., Lee, S., Malone, A.M., Paul, B.P., Jr., and Woodard, B.S., "Ice-Accretion Test Results for Three Large-Scale Swept-Wing Models in the NASA Icing Research Tunnel,” AIAA Paper 2016-3733, June 2016; also NASA/TM-2016-219137, Sept. 2016.

[5] Camarinha Fujiwara, G., "Design of 3D swept wing hybrid models for icing wind tunnel tests.", 2015, M.S. Thesis, Department of Aerospace Engineering, University of Illinois at Urbana-Champaign.

doi: http://hdl.handle.net/2142/72880

[6] Fujiwara, G. E., Woodard, B., Wiberg, B., Mortonson, A. J., and Bragg, M., "A Hybrid Airfoil Design Method for Icing Wind Tunnel Tests", 5th AIAA Atmospheric and Space Environments Conference, AIAA-2013-2826, June 2013.

doi: $10.2514 / 6.2013-2826$

[7] Fujiwara, G. E., Wiberg, B. D., Woodard, B., and Bragg, M., "3D Swept Hybrid Wing Design Method for Icing Wind Tunnel Tests", 6th AIAA Atmospheric and Space Environments Conference, AIAA-2014-2616, June 2014

doi: $10.2514 / 6.2014-2616$

[8] Wiberg, B. D., Fujiwara, G. E., Woodard, B., and Bragg, M., "Large-Scale Swept-Wing Icing Simulations in the NASA Glenn Icing Research Tunnel Using LEWICE3D", 6th AIAA Atmospheric and Space Environments Conference, AIAA-20142617, June 2014.

doi: $10.2514 / 6.2014-2617$

[9] Camello, S. C., Lee, S., Lum, C. W., and Bragg, M. B., "Generation of Fullspan Leading-Edge 3D Ice Shapes for SweptWing Aerodynamic Testing," Proceedings of the 8th AIAA Atmospheric and Space Environments Conference, AIAA Paper 20163737, Washington D.C., June 2016.

[10] Broeren, A.P., Woodard, B.S., Diebold, J.M., and Moens, F., "Low-Reynolds Number Aerodynamics of an $8.9 \%$ Scale Semispan Swept Wing for Assessment of Icing Effects,” AIAA Paper 2017-4372; also NASA/TM-2017-219533, July 2017.

[11] Camello, S.C., Bragg, M.B., Broeren, A.P., Lum, C.W., Woodard, B.S., and Lee, S., "Effect of Ice Shape Fidelity on SweptWing Aerodynamic Performance,” AIAA Paper 2017-4373, June 2017.

[12] Lum, C.W., Sandhu, N., Diebold, J.M., Woodard, B.S., and Bragg, M.B., "The Application of a Five-Hole Probe WakeSurvey Technigue to the Study of Swept Wing Icing Aerodynamics,” AIAA Paper 2017-4374, June 2017. 
[13] Lynch, F. T., and Khodadoust, A., "Effects of Ice Accretions on Aircraft Aerodynamics," Progress in Aerospace Sciences, Volume 37, Issue 8, Nov. 2001, pp. 669-767.

doi: $10.1016 / \mathrm{S} 0376-0421(01) 00018-5$

[14] Poll, D.I.A., "Spiral Vortex Flow Over a Swept-back Wing,” Aeronautical Journal, May 1986, pp.185-199.

[15] Broeren, A.P., Bragg, M. B., Addy, H. E., Lee, S., Moens, F., and Guffond, Didier, "Effect of High-Fidelity Ice Accretion Simulations on the Performance of a Full-Scale Airfoil Model," AIAA Paper 2008-434; also NASA/TM-2010-216344, June 2010 .

[16] Diebold, J. M., Monastero, M. C., and Bragg, M. B., “Aerodynamics of a Swept Wing with Ice Accretion at Low Reynolds Number," AIAA Paper 2012-2795

[17] Broeren, A. P., Woodard, B. S., Diebold, J. M., and Bragg, M. B., "Preliminary Testing of Low-Reynolds Number Aerodynamics for a Swept Wing with Artificial Ice Roughness," DOT/FAA/TC-17/48, 2017.

[18] Lee, S., Broeren, A. P., Woodard, B. S., Lum, C. W., Smith, T. G., "Comparison of Iced Aerodynamic Measurements on Swept Wing from Two Wind Tunnels," AIAA 10th Atmospheric and Space Environments Conference, Atlanta, GA, June 25-29, 2018 (submitted for publication), 2018.

[19] Broeren, A. P., Lee, S., Woodard, B. S., Lum, C. W., Smith, T. G., "Independent Effects of Reynolds and Mach Numbers on the Aerodynamics of an Iced Swept Wing," AIAA 10th Atmospheric and Space Environments Conference, Atlanta, GA, June 25-29, 2018 (submitted for publication), 2018.

[20] Kerho, M. F., Bragg, M. B., and Khodadoust, A., "LDV Flowfield Measurements on a Straight and Swept Wing with a Simulated Ice Accretion," AIAA Paper 1993-0300

[21] Coleman, H.W., and Steele, W.G., Experimentation and Uncertainty Analysis for Engineers, Wiley-Interscience, New York, 1989, pp.40-118.

[22] Kline, S., and McClintock, F.A, "Describing Uncertainties in Single Sample Experiments," Mechanical Engineering, Vol. 75, No. 1, 1953, pp. 3-8. 